\title{
Reed-Solomon Coded Cooperative Spatial Modulation Based on Nested Construction for Wireless Communication
}

\author{
Chunli ZHAO, Fengfan YANG, Daniel Kariuki WAWERU \\ College of Electronics and Information Engg., Nanjing Univ. of Aeronautics and Astronautics, Nanjing, 210016 China \\ chunlizhao_cn@163.com, yang_fengfan@vip.sina.com, daniel.kariuki19@nuaa.edu.cn \\ Submitted November 7, 2020 / Accepted February 14, 2021
}

\begin{abstract}
This paper proposes the Reed-Solomon coded cooperative spatial modulation (RSCC-SM) scheme based on nested construction of two Reed-Solomon (RS) codes over quasi-static Rayleigh fading channel. In this construction, the RS code with larger number of consecutive roots is employed at the relay node while the RS code with less number of consecutive roots is employed at the source node. The $R S$ code with excessive roots at the relay node offers some extra redundancy. The enhanced bit error rate $(B E R)$ performance will be obtained if source and relay $R S$ codes are jointly decoded at the destination node. Therefore, the authors propose the joint RS decoding based on two different approaches known as naive approach and smart approach. Monte Carlo simulated results reveal that the proposed RSCC-SM scheme utilizing smart approach not only outperforms its corresponding coded non-cooperative scheme but also outperforms its counterpart RSCCSM scheme employing naive approach under identical conditions.
\end{abstract}

\section{Keywords}

Reed-Solomon (RS) code, joint RS decoding, nested construction, coded cooperation, Spatial Modulation (SM)

\section{Introduction}

Multiple-input multiple-output (MIMO) systems have been recognized as a core technique for improving the spectral efficiency and link reliability comparing with single antenna systems [1]. A variety of MIMO transmission techniques have been introduced and widely used in many wireless standards. A well-known MIMO technique is the vertical Bell Labs layered space-time (V-BLAST) architecture [2]. However, simultaneously transmitting multiple independent data streams from all the antennas require the inter-antenna synchronization (IAS). The VBLAST scheme also suffers from the inter-channel interference (ICI) at the receiver. A recently developed tech- nique called spatial modulation (SM) can effectively alleviate the ICI and IAS [3]. This is because only one transmit antenna is activated to convey constellation symbol at each time instant. Moreover, the utilization of the transmit antenna index as an information-bearing unit effectively boosts the spectral efficiency [4].

A typical cooperative communication system contains three nodes usually named as source, relay and destination nodes [5]. Various cooperative protocols such as decodeand-forward (DF), compress-and-forward (CF) and amplify-and-forward (AF) have been proposed [6], [7]. The authors in [8] proposed the idea of coded cooperation as an integration of channel codes and cooperative protocols, which can effectively combat channel impairments. In coded cooperation, the coded sequence is usually divided into two parts. One part is transmitted by the source node, and the rest is transmitted by the relay node. At the destination node, we use the joint decoding to retrieve the source transmitted signal. Recently, the coded cooperation has drawn substantial research efforts. Many kinds of channel codes have been employed in coded cooperation. In [9], the authors applied the quasi-cyclic low-density parity-check (QC-LDPC) code in cooperative communication, then proposed the QC-LDPC coded cooperative scheme. In [10], the asymmetric turbo coded cooperative scheme as an integration of asymmetric turbo code and cooperative protocol was introduced, where code matched interleaver (CMI) and multiple relays are employed in the proposed scheme. Moreover, the polar coded cooperative scheme with Plotkin's construction was proposed in [11].

However, most of the aforementioned coded cooperative schemes focus on binary codes. The coded cooperative schemes for non-binary channel codes are not widely investigated. As a class of non-binary codes, the famous Reed-Solomon (RS) codes have low encoding and decoding complexity. Moreover, RS codes are maximum distance separable (MDS) codes and play a very effective role in correcting random burst errors [12]. This brings motivation to explore RS codes, which are employed in cooperative scheme to efficiently improve the bit error rate (BER) performance of coded cooperation system. In [13], the 
combination of RS codes and convolution codes was utilized in the coded cooperation, which yields reasonable BER performance. In [14], the authors investigated the coded cooperative scheme of RS codes to determine the level of cooperation. Coded cooperation using RS codes in slow fading channel was reported in [15]. However, the RS coded cooperative scheme with SM has not been probed to the best of our knowledge. As we all know, high spectral efficiency is one key factor driving the development of future wireless communications. Therefore, it is very necessary to further improve the spectral efficiency of the coded cooperative system. Many available literatures (such as the literature $[2,6,16])$ have also reported that the combination of SM with cooperation scheme can enhance the spectral efficiency of the system. Motivated by this, the RS coded cooperative SM (RSCC-SM) scheme as an integration of RS coded cooperation and SM is proposed in this paper. In the RSCC-SM scheme, two different RS codes are placed at the source and relay nodes, respectively. The RS code utilized at the relay node has relatively more parity-check symbols as compared to the RS code placed at the source node, and possesses more error correcting capability. Therefore, a part of source transmitted message sequence is re-encoded at the relay node. In order to improve error performance, the joint RS decoding is established at the destination which jointly decodes the source and relay signal. In this paper, two joint decoding approaches i.e., naive approach and smart approach are proposed. Moreover, a non-cooperative RS coded SM (RSCSM) scheme is designed for benchmark purpose.

The contributions of this paper are listed as follows:

- The RSCC-SM scheme based on nested construction (two RS codes) through quasi-static Rayleigh fading channel is proposed.

- The RSC-SM scheme through quasi-static Rayleigh fading channel in non-cooperative scenario is developed as the benchmark to RSCC-SM scheme.

- The joint RS decoding is established for RSCC-SM scheme based on two different approaches, i.e., naive approach and smart approach.

- The BER performance of the proposed RSCC-SM scheme is also evaluated in practical scenarios, i.e., non-ideal source-to-relay link.

The remaining structure of this paper is organized as follows. The nested construction of RS code is introduced in Sec. 2. The RSCC-SM scheme is presented in Sec. 3. Section 4 discusses the RSC-SM scheme. Section 5 demonstrates the joint RS decoding. The simulation results are shown in Sec. 6. Finally, Section 7 concludes this manuscript.

\section{Nested Construction of RS Code}

The RS code is a non-binary maximum distance separable (MDS) code, which is a special type of MDS code. This non-binary code reaches the singleton bound and

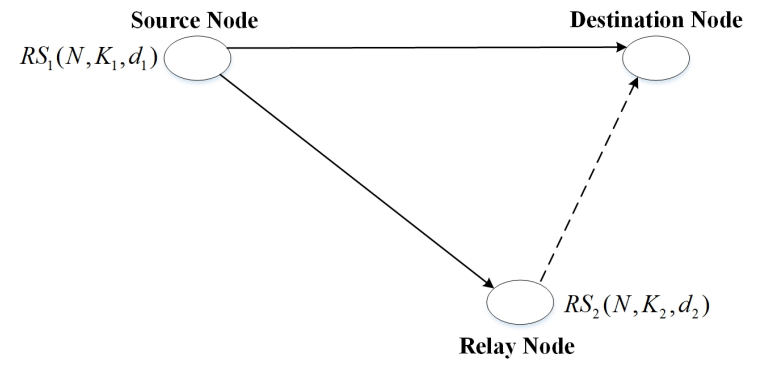

Fig. 1. Nested construction of RS code.

achieves the maximum distance $[12,17]$. In order to get the improved error performance in cooperative scenario, two $\mathrm{RS}$ codes can be arranged in nested/cooperative form as shown in Fig. 1.

In this construction, the RS code $R S_{1}\left(N, K_{1}, d_{1}\right)$ with large number of consecutive roots is employed at the relay node while the other RS code $R S_{2}\left(N, K_{2}, d_{2}\right)$ with small number of consecutive roots is employed at the source node. The RS code $R S_{j}\left(N, K_{j}, d_{j}\right)$ for $j=1,2$ has a code rate $R_{j}=K_{j} / N$ where the parameters $N, K_{j}$ and $d_{j}$ denote the codeword length, code dimension and minimum distance, respectively. Since RS code is an MDS code, the minimum distance $d_{j}$ of $R S_{j}\left(N, K_{j}, d_{j}\right)$ is exactly equal to $d_{j}=N-K_{j}+1$. Furthermore, the generator polynomials of $R S_{j}\left(N, K_{j}, d_{j}\right)$ with $N-K_{j}$ consecutive roots are represented as

$$
\begin{aligned}
\mathbf{g}_{1}(X) & =(X-\beta)\left(X-\beta^{2}\right) \ldots\left(X-\beta^{N-K_{1}}\right) \\
& =g_{0}^{(1)}+g_{1}^{(1)} X+\ldots+g_{N-K_{1}}^{(1)} X^{N-K_{1}}, \\
\mathbf{g}_{2}(X) & =(X-\gamma)\left(X-\gamma^{2}\right) \ldots\left(X-\gamma^{N-K_{2}}\right) \\
& =g_{0}^{(2)}+g_{1}^{(2)} X+\ldots+g_{N-K_{2}}^{(2)} X^{N-K_{2}}
\end{aligned}
$$

where $\beta^{f^{\prime}} \in \mathrm{GF}\left(2^{n}\right) \quad(n=1,2, \ldots)$ are the roots of $\mathbf{g}_{1}(X)$ and $g_{f_{1}}^{(1)} \in \mathrm{GF}\left(2^{n}\right)$ are the coefficients of $\mathbf{g}_{1}(X)$ for $f_{1}^{\prime} \in\left\{1,2, \ldots, N-K_{1}\right\}$ and $f_{1} \in\left\{0,1, \ldots, N-K_{1}\right\}$. Similarly, $\gamma^{f_{2}^{\prime}} \in \mathrm{GF}\left(2^{n}\right)$ are the roots of $\mathbf{g}_{2}(X)$ and $g_{f_{2}}^{(2)} \in \mathrm{GF}\left(2^{n}\right)$ are the coefficients of $\mathbf{g}_{2}(X)$ for $f_{2}^{\prime} \in\left\{1,2, \ldots, N-K_{2}\right\}$ and $f_{2} \in\left\{0,1, \ldots, N-K_{2}\right\}$. As $K_{1}>K_{2}$, therefore the generator polynomial $\mathbf{g}_{2}(X)$ of RS code $R S_{2}\left(N, K_{2}, d_{2}\right)$ comprises of more consecutive roots than $\mathbf{g}_{1}(X)$ and has better error correcting capability [17]. The encoded sequences of the source and relay nodes are jointly decoded by joint RS decoder at the destination node, which will be discussed in Sec. 5.

\section{RS Coded Cooperative SM (RSCC- SM) Scheme}

Figure 2 demonstrates the RSCC-SM scheme, which is based on the three-terminal communication idea of Van der Meulen [5]. In the RSCC-SM scheme, the source and relay nodes possess an identical number of antennas denoted as $N_{\mathrm{T}}$. At the destination node, the number of receive antennas is denoted as $N_{\mathrm{R}}$. Different from paper [5], our proposed scheme introduces the interesting concept of 


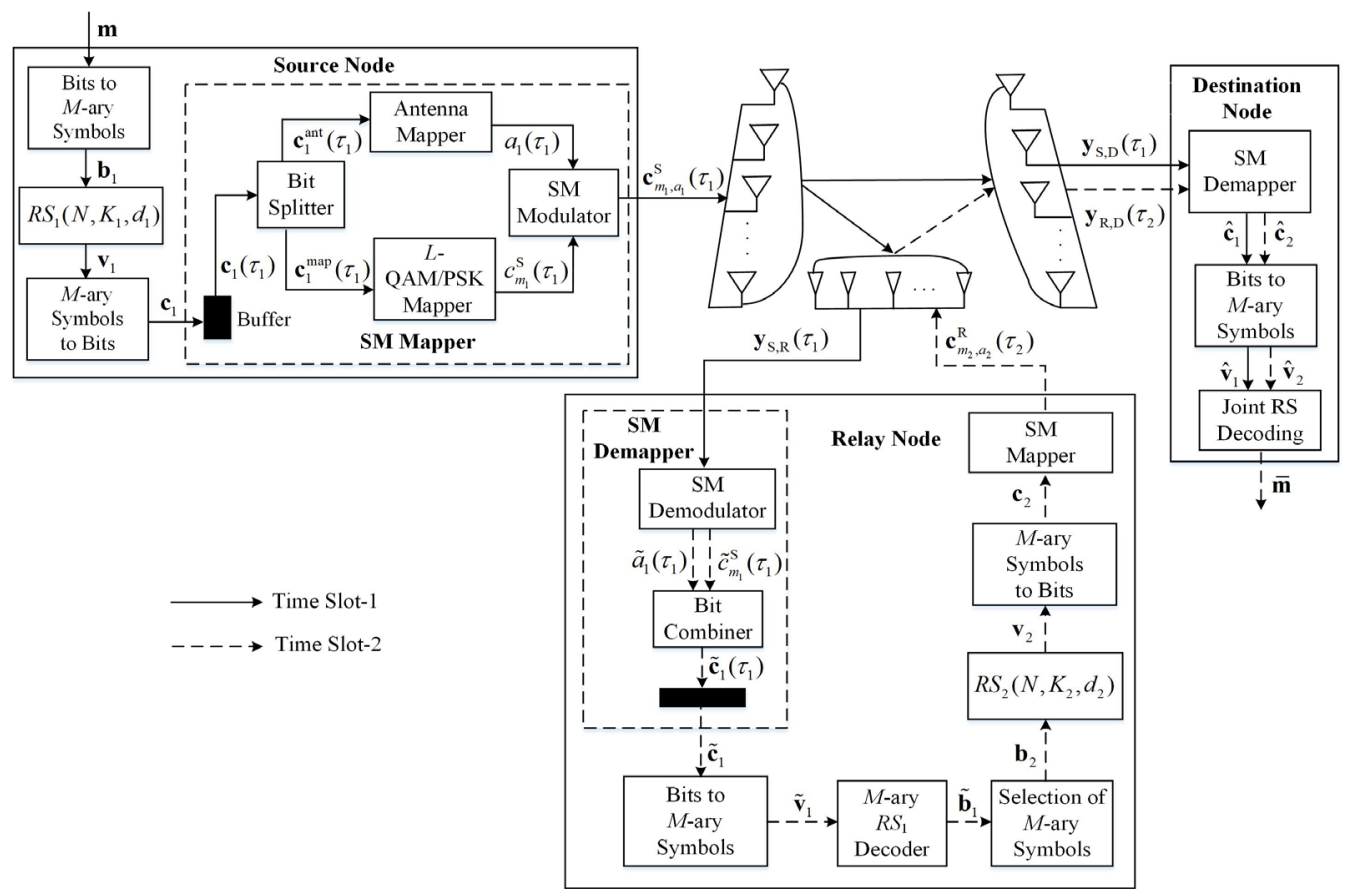

Fig. 2. RSCC-SM scheme for cooperative communication.

information selection at the relay node. The joint RS decoder is used at the destination to jointly decode the source and relay signal. Moreover, the emerging SM technique is utilized in the three-terminal communication network to further boost the spectral efficiency of cooperative system. There are several relay protocols such as CF, AF and DF. In the proposed coded cooperative scheme, the DF relaying protocol is considered and an entire communication transmission requires two time slots.

During time slot-1, the source node maps the binary information sequence $\mathbf{m}$ into non-binary $M$-ary symbol sequence $\mathbf{b}_{1}$, which are the symbols in $\operatorname{GF}\left(2^{n}\right)$ $(n=1,2, \ldots)$. The non-binary information sequence $\mathbf{b}_{1}$ is encoded by the systematic RS code $R S_{1}\left(N, K_{1}, d_{1}\right)$ employing the generator polynomial $\mathbf{g}_{1}(X)$ shown in (1). Then, the codeword polynomial $\mathbf{v}_{1}(X)$ of systematic codeword $\mathbf{v}_{1}$ can be expressed as

$$
\mathbf{v}_{1}(X)=X^{N-K_{1}} \mathbf{b}_{1}(X)+\mathbf{p}_{1}(X)
$$

where $\mathbf{b}_{1}(X)=b_{0}^{(1)}+b_{1}^{(1)} X+\ldots+b_{K_{1}-1}^{(1)} X^{K_{1}-1}\left(b_{i_{1}}^{(1)} \in \mathrm{GF}\left(2^{n}\right)\right.$ and $\left.i_{1} \in\left\{0,1, \ldots, K_{1}-1\right\}\right)$ is the polynomial representation of information symbol sequence $\mathbf{b}_{1}$, and the polynomial $\mathbf{v}_{1}(X)=v_{0}^{(1)}+v_{1}^{(1)} X+\ldots+v_{N-1}^{(1)} X^{N-1}$ for $v_{l_{1}}^{(1)} \in \mathrm{GF}\left(2^{n}\right)$ and $l_{1} \in\{0,1, \ldots, N-1\}$ is the codeword polynomial. Moreover, the polynomial $\mathbf{p}_{1}(X)=p_{0}^{(1)}+p_{1}^{(1)} X+\ldots+p_{N-K_{1}-1}^{(1)} X^{N-K_{1}-1}$ $\left(p_{j_{1}}^{(1)} \in \mathrm{GF}\left(2^{n}\right)\right.$ and $\left.j_{1} \in\left\{0,1, \ldots, N-K_{1}-1\right\}\right)$ represents the parity polynomial that is computed as follows

$$
\mathbf{p}_{1}(X)=X^{N-K_{1}} \mathbf{b}_{1}(X) / \mathbf{g}_{1}(X) .
$$

The codeword polynomial $\mathbf{v}_{1}(X)$ can be represented in sequence form i.e., $\mathbf{v}_{1}=\left[v_{0}^{(1)}, v_{1}^{(1)}, \ldots, v_{N-1}^{(1)}\right]=\left[\mathbf{p}_{1}, \mathbf{b}_{1}\right]$, where $\mathbf{p}_{1}=\left[p_{0}^{(1)}, p_{1}^{(1)}, \ldots, p_{N-K_{1}-1}^{(1)}\right]$ and $\mathbf{b}_{1}=\left[b_{0}^{(1)}, b_{1}^{(1)}, \ldots, b_{K_{1}-1}^{(1)}\right]$ are the parity and message symbol sequences, respectively.
After the RS encoding, the non-binary codeword $\mathbf{v}_{1}$ is generated. With regard to the codeword symbol sequence $\mathbf{v}_{1}$, each symbol $v_{l_{1}}^{(1)}$ can be denoted as a binary vector $\mathbf{v}^{\prime}=\left[v_{0}^{\prime}, v_{1}^{\prime}, \ldots, v_{n-1}^{\prime}\right]$ having length $n$, where $v_{i}^{\prime} \in\{0,1\}$ and $i \in\{0,1, \ldots, n-1\}$. Through the ' $M$-ary symbols to bits' block, the binary sequence $\mathbf{c}_{1}$ is generated as demonstrated in Fig. 2. Then, the sequence $\mathbf{c}_{1}$ is divided into short sequences $\mathbf{c}_{1}\left(\tau_{1}\right)$ of length $n=\log _{2}\left(N_{\mathrm{T}}\right)+\log _{2}(L)$ by buffer, where $\tau_{1} \in\{1,2, \ldots, N\}$ and $L$ denotes the modulation order. Through the bit splitter, the generated sequence $\mathbf{c}_{1}\left(\tau_{1}\right)$ is further separated into two parts $\mathbf{c}_{1}^{\text {ant }}\left(\tau_{1}\right)$ and $\mathbf{c}_{1}^{\text {map }}\left(\tau_{1}\right)$. The first part $\mathbf{c}_{1}^{\text {ant }}\left(\tau_{1}\right)$ with length $\log _{2}\left(N_{\mathrm{T}}\right)$ is given into the antenna mapper to select one active transmit antenna index labelled by $a_{1}\left(\tau_{1}\right) \in\left\{1,2, \ldots, N_{\mathrm{T}}\right\}$. The remaining part $\mathbf{c}_{1}^{\text {map }}\left(\tau_{1}\right)$ is sent to the $L$-QAM/PSK mapper that outputs the modulated symbol $c_{m_{1}}^{\mathrm{S}}\left(\tau_{1}\right)$, where $m_{1} \in\{1,2, \ldots, L\}$. The SM modulator puts the symbol $c_{m_{1}}\left(_{1}\right)$ to the $a_{1}\left(\tau_{1}\right)$ th transmit antenna. Accordingly, the $N_{\mathrm{T}} \times 1$ transmission vector $\mathbf{c}_{m_{1}, a_{1}}\left(\tau_{1}\right)$ is generated as follows

$$
\mathbf{c}_{m_{1}, a_{1}}^{\mathrm{S}}\left(\tau_{1}\right)=\left[\ldots, 0, c_{m_{1}}^{\mathrm{S}}\left(\tau_{1}\right), 0, \ldots\right]^{\mathrm{T}}
$$

where $[\cdot]^{\mathrm{T}}$ represents the transpose of a vector or matrix. Note that buffer, bit splitter, antenna mapper, $L$-QAM/PSK mapper and SM modulator form the SM mapper block as depicted in Fig. 2. For illustration, Table 1 exhibits different mapping results of SM scheme with code over $\mathrm{GF}\left(2^{4}\right)$, where the product of the number of transmit antennas $N_{\mathrm{T}}$ and modulation order $L$ is equal to 16 , i.e., $L N_{\mathrm{T}}=16$. In Tab. 1 , the notation $\alpha$ is a primitive element of $\mathrm{GF}\left(2^{4}\right)$. Accordingly, $\alpha^{-\infty} \triangleq 0, \alpha^{0}=1, \alpha, \ldots, \alpha^{14}$ constitute all the elements of $\mathrm{GF}\left(2^{4}\right)$. In fact, various finite fields possess different communication scenarios under various combinations of $N_{\mathrm{T}}$ and $L$. These communication scenarios 


\begin{tabular}{|c|c|c|c|c|}
\hline \multirow{2}{*}{$\begin{array}{c}\text { Field } \\
\text { elements }\end{array}$} & \multirow{2}{*}{$\begin{array}{l}\text { Binary } \\
\text { vector }\end{array}$} & $N_{\mathrm{T}}=2,8-\mathrm{QAM}$ & $N_{\mathrm{T}}=4,4-\mathrm{QAM}$ & $N_{\mathrm{T}}=8, \mathrm{BPSK}$ \\
\hline & & Antenna index, modulated symbol & Antenna index, modulated symbol & Antenna index, modulated symbol \\
\hline 0 & 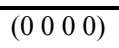 & $(1,-3+i)$ & $(1,-1-i)$ & $(1,-1)$ \\
\hline 1 & 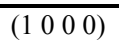 & $(2,-3+\mathrm{i})$ & $(3,-1-i)$ & $(5,-1)$ \\
\hline$\alpha$ & $\left(\begin{array}{llll}0 & 1 & 0 & 0\end{array}\right)$ & $(1,3+\mathrm{i})$ & $(2,-1-i)$ & $(3,-1)$ \\
\hline$\alpha^{2}$ & $\left(\begin{array}{llllll}0 & 0 & 1 & 0\end{array}\right)$ & $(1,-1+\mathrm{i})$ & $(1,1-\mathrm{i})$ & $(2,-1)$ \\
\hline$\alpha^{3}$ & $\left.\begin{array}{lllll}(0 & 0 & 0 & 1\end{array}\right)$ & $(1,-3-i)$ & $(1,-1+i)$ & $(1,1)$ \\
\hline$\alpha^{4}$ & $\left(\begin{array}{lllllllll}1 & 1 & 0 & 0\end{array}\right)$ & $(2,3+\mathrm{i})$ & $(4,-1-i)$ & $(7,-1)$ \\
\hline$\alpha^{5}$ & $\left(\begin{array}{llllll}0 & 1 & 1 & 0\end{array}\right)$ & $(1,1+\mathrm{i})$ & $(2,1-i)$ & $(4,-1)$ \\
\hline$\alpha^{6}$ & $\left(\begin{array}{llll}0 & 0 & 1 & 1\end{array}\right)$ & $(1,-1-i)$ & $(1,1+\mathrm{i})$ & $(2,1)$ \\
\hline$\alpha^{7}$ & $\left(\begin{array}{llll}1 & 1 & 0 & 1\end{array}\right)$ & $(2,3-i)$ & $(4,-1+i)$ & $(7,1)$ \\
\hline$\alpha^{8}$ & $\left(\begin{array}{lllllllllll}1 & 0 & 1 & 0\end{array}\right)$ & $(2,-1+i)$ & $(3,1-\mathrm{i})$ & $(6,-1)$ \\
\hline$\alpha^{9}$ & $\left(\begin{array}{llll}0 & 1 & 0 & 1\end{array}\right)$ & $(1,3-i)$ & $(2,-1+i)$ & $(3,1)$ \\
\hline$\alpha^{10}$ & $\left(\begin{array}{llll}1 & 1 & 1 & 0\end{array}\right)$ & $(2,1+\mathrm{i})$ & $(4,1-i)$ & $(8,-1)$ \\
\hline$\alpha^{11}$ & $\left.\begin{array}{llllll} & 0 & 1 & 1 & 1\end{array}\right)$ & $(1,1-i)$ & $(2,1+\mathrm{i})$ & $(4,1)$ \\
\hline$\alpha^{12}$ & $\left(\begin{array}{llll}1 & 1 & 1 & 1\end{array}\right)$ & $(2,1-i)$ & $(4,1+i)$ & $(8,1)$ \\
\hline$\alpha^{13}$ & $\left(\begin{array}{llll}1 & 0 & 1 & 1\end{array}\right)$ & $(2,-1-i)$ & $(3,1+\mathrm{i})$ & $(6,1)$ \\
\hline$\alpha^{14}$ & $\left(\begin{array}{llll}1 & 0 & 0 & 1\end{array}\right)$ & $(2,-3-i)$ & $(3,-1+i)$ & $(5,1)$ \\
\hline
\end{tabular}

Tab. 1. Different mapping results of SM scheme with code over $\mathrm{GF}\left(2^{4}\right)$.

\begin{tabular}{|c|c|c|}
\hline Finite field & $N_{\mathrm{T}}$ & $L$ \\
\hline \multirow{2}{*}{$\mathrm{GF}\left(2^{3}\right)$} & 2 & 4 \\
\hline & 4 & 2 \\
\hline \multirow{3}{*}{$\mathrm{GF}\left(2^{4}\right)$} & 2 & 8 \\
\hline & 4 & 4 \\
\hline & 8 & 2 \\
\hline \multirow{4}{*}{$\mathrm{GF}\left(2^{5}\right)$} & 2 & 16 \\
\hline & 4 & 8 \\
\hline & 8 & 4 \\
\hline & 16 & 2 \\
\hline \multirow{5}{*}{$\operatorname{GF}\left(2^{6}\right)$} & 2 & 32 \\
\hline & 4 & 16 \\
\hline & 8 & 8 \\
\hline & 16 & 4 \\
\hline & 32 & 2 \\
\hline \multirow{6}{*}{$\mathrm{GF}\left(2^{7}\right)$} & 2 & 64 \\
\hline & 4 & 32 \\
\hline & 8 & 16 \\
\hline & 16 & 8 \\
\hline & 32 & 4 \\
\hline & 64 & 2 \\
\hline \multirow{7}{*}{$\mathrm{GF}\left(2^{8}\right)$} & 2 & 128 \\
\hline & 4 & 64 \\
\hline & 8 & 32 \\
\hline & 16 & 16 \\
\hline & 32 & 8 \\
\hline & 64 & 4 \\
\hline & 128 & 2 \\
\hline
\end{tabular}

Tab. 2. Different communication scenarios with RS code over various Galois fields.

with RS code over $\mathrm{GF}\left(2^{3}\right), \mathrm{GF}\left(2^{4}\right), \mathrm{GF}\left(2^{5}\right), \mathrm{GF}\left(2^{6}\right)$, $\mathrm{GF}\left(2^{7}\right)$ and $\mathrm{GF}\left(2^{8}\right)$ are shown in Tab. 2.

The transmission vector $\mathbf{c}_{m_{1}, a_{1}}^{\mathrm{S}}\left(\tau_{1}\right)$ is then sent to relay and destination nodes through Rayleigh fading channel, respectively. The received vector at the relay node is mathematically written as

$$
\begin{aligned}
\mathbf{y}_{\mathrm{S}, \mathrm{R}}\left(\tau_{1}\right) & =\mathbf{H}_{\mathrm{S}, \mathrm{R}} \mathbf{c}_{m_{1}, a_{1}}^{\mathrm{S}}\left(\tau_{1}\right)+\mathbf{n}_{\mathrm{S}, \mathrm{R}}\left(\tau_{1}\right) \\
& =\mathbf{h}_{\mathrm{S}, \mathrm{R}}^{a_{1}\left(\tau_{1}\right)} c_{m_{1}}^{\mathrm{S}}\left(\tau_{1}\right)+\mathbf{n}_{\mathrm{S}, \mathrm{R}}\left(\tau_{1}\right)
\end{aligned}
$$

where $\mathbf{H}_{\mathrm{S}, \mathrm{R}}$ denotes the $N_{\mathrm{T}} \times N_{\mathrm{T}}$ quasi-static Rayleigh fading channel matrix and $\mathbf{h}_{\mathrm{S}, \mathrm{R}}^{a_{1}\left(\tau_{1}\right)}$ represents the $a_{1}\left(\tau_{1}\right)$ th column vector of $\mathbf{H}_{\mathrm{S}, \mathrm{R}}$. The entries of $\mathbf{H}_{\mathrm{S}, \mathrm{R}}$ are independent identically distributed (i.i.d.) complex random variables that follow Gaussian distribution $\mathrm{CN}(0,1)$. $\mathbf{n}_{\mathrm{S}, \mathrm{R}}\left(\tau_{1}\right)$ represents the $N_{\mathrm{T}} \times 1$ additive Gaussian noise vector. The entries of $\mathbf{n}_{\mathrm{S}, \mathrm{R}}\left(\tau_{1}\right)$ are independent and have the complex Gaussian distribution $\mathrm{CN}\left(0, \delta^{2}\right)$, where $\delta^{2}$ is the power spectral density of noise.

During the same time slot-1, the relay node employs the SM demodulator to perform joint maximum-likelihood (ML) detection for active transmit antenna index $a_{1}\left(\tau_{1}\right)$ and modulated symbol $c_{m_{1}}^{\mathrm{s}}\left(\tau_{1}\right)$. The estimated transmit antenna index $\tilde{a}_{1}\left(\tau_{1}\right)$ and modulated symbol $\tilde{c}_{m_{1}}^{\mathrm{S}}\left(\tau_{1}\right)$ are combined into $\tilde{\mathbf{c}}_{1}\left(\tau_{1}\right)$, which is buffered to get the estimated binary codeword sequence $\tilde{\mathbf{c}}_{1}$. After that, the binary sequence $\tilde{\mathbf{c}}_{1}$ is mapped to non-binary sequence $\tilde{\mathbf{v}}_{1}$. The $\mathrm{SM}$ demapper is comprised of the SM demodulator, bit combiner and buffer as illustrated in Fig. 2.

During time slot-2, the $M$-ary $R S_{1}$ decoder gets $\tilde{\mathbf{v}}_{1}$ and generates $\tilde{\mathbf{b}}_{1}$ that is used as an estimate of information symbol sequence $\mathbf{b}_{1}$. The Euclidean decoding algorithm is used by the $M$-ary $R S_{1}$ decoder. The estimated non-binary message sequence $\tilde{\mathbf{b}}_{1}$ is then partially encoded by $R S_{2}\left(N, K_{2}, d_{2}\right)$ using the generator polynomial $\mathbf{g}_{2}(X)$ 
given in (2). The systematic encoding of $R S_{2}\left(N, K_{2}, d_{2}\right)$ is defined as

$$
\mathbf{v}_{2}(X)=X^{N-K_{2}} \mathbf{b}_{2}(X)+\mathbf{p}_{2}(X)
$$

where $\mathbf{v}_{2}(X)=v_{0}^{(2)}+v_{1}^{(2)} X+\ldots+v_{N-1}^{(2)} X^{N-1}$ for $v_{l_{2}}^{(2)} \in \mathrm{GF}\left(2^{n}\right)$ and $l_{2} \in\{0,1, \ldots, N-1\}$ is the codeword polynomial at the relay node. $\mathbf{b}_{2}(X)=b_{0}^{(2)}+b_{1}^{(2)} X+\ldots+b_{K_{2}-1}^{(2)} X^{K_{2}-1}$ is the message polynomial at the relay node and can be represented as sequence $\mathbf{b}_{2}=\left[b_{0}^{(2)}, b_{1}^{(2)}, \ldots, b_{K_{2}-1}^{(2)}\right]$, where $b_{i}^{(2)} \in \mathrm{GF}\left(2^{n}\right)$ and $i_{2} \in\left\{0,1, \ldots, K_{2}-1\right\}$. The relay node selects the first $K_{2}$ symbols from the estimated non-binary message sequence $\tilde{\mathbf{b}}_{1}$ as the relay message sequence for the partial encoding such as $\mathbf{b}_{2}=\left[\tilde{b}_{0}^{(1)}, \tilde{b}_{1}^{(1)}, \ldots, \tilde{b}_{K_{2}-1}^{(1)}\right]$. Similarly, as given in (4) the parity polynomial for the RS code $R S_{2}\left(N, K_{2}, d_{2}\right)$ can also be calculated as follows

$$
\mathbf{p}_{2}(X)=X^{N-K_{2}} \mathbf{b}_{2}(X) / \mathbf{g}_{2}(X)
$$

where $\mathbf{p}_{2}(X)=p_{0}^{(2)}+p_{1}^{(2)} X+\ldots+p_{N-K_{2}-1}^{(2)} X^{N-K_{2}-1}$ for $p_{j_{2}}^{(2)} \in \mathrm{GF}\left(2^{n}\right)$ and $j_{2} \in\left\{0,1, \ldots, N-K_{2}-1\right\}$. The parity symbol sequence $\mathbf{p}_{2}$ can be represented as $\mathbf{p}_{2}=\left[p_{0}^{(2)}, p_{1}^{(2)}, \ldots, p_{N-K_{2}-1}^{(2)}\right]$. The codeword polynomial $\mathbf{v}_{2}(X)$ can be represented in sequence form i.e., $\mathbf{v}_{2}=\left[v_{0}^{(2)}, v_{1}^{(2)}, \ldots, v_{N-1}^{(2)}\right]=\left[p_{0}^{(2)}, p_{1}^{(2)}, \ldots, p_{N-K_{2}-1}^{(2)}, b_{0}^{(2)}, b_{1}^{(2)}, \ldots, b_{K_{2}-1}^{(2)}\right]$. The non-binary codeword sequence $\mathbf{v}_{2}$ of RS code $R S_{2}\left(N, K_{2}, d_{2}\right)$ is obtained by the use of (7) and it is further converted into binary sequence $\mathbf{c}_{2}$. After the SM mapper, the transmission vector $\mathbf{c}_{m_{2}, a_{2}}^{\mathrm{R}}\left(\tau_{2}\right)$ is generated as

$$
\mathbf{c}_{m_{2}, a_{2}}^{\mathrm{R}}\left(\tau_{2}\right)=\left[\ldots, 0, c_{m_{2}}^{\mathrm{R}}\left(\tau_{2}\right), 0, \ldots\right]^{\mathrm{T}}
$$

where $c_{m_{2}}^{\mathrm{R}}\left(\tau_{2}\right)$ is the $L$-QAM/PSK symbol transmitted at the $a_{2}\left(\tau_{2}\right)$ th transmit antenna, $m_{2} \in\{1,2, \ldots, L\}$, $a_{2}\left(\tau_{2}\right) \in\left\{1,2, \ldots, N_{\mathrm{T}}\right\}$ and $\tau_{2} \in\{1,2, \ldots, N\}$. The relay node then propagates the transmission vector $\mathbf{c}_{m_{2}, a_{2}}^{\mathrm{R}}\left(\tau_{2}\right)$ to destination node through Rayleigh fading channel.

During their respective time slots, the destination node receives the signal vectors denoted as

$$
\begin{aligned}
\mathbf{y}_{\mathrm{S}, \mathrm{D}}\left(\tau_{1}\right) & =\mathbf{H}_{\mathrm{S}, \mathrm{D}} \mathbf{c}_{m_{1}, a_{1}}^{\mathrm{S}}\left(\tau_{1}\right)+\mathbf{n}_{\mathrm{S}, \mathrm{D}}\left(\tau_{1}\right) \\
& =\mathbf{h}_{\mathrm{S}, \mathrm{D}}^{a_{1}\left(\tau_{1}\right)} c_{m_{1}}^{\mathrm{S}}\left(\tau_{1}\right)+\mathbf{n}_{\mathrm{S}, \mathrm{D}}\left(\tau_{1}\right), \\
\mathbf{y}_{\mathrm{R}, \mathrm{D}}\left(\tau_{2}\right) & =\mathbf{H}_{\mathrm{R}, \mathrm{D}} \mathbf{c}_{m_{2}, a_{2}}^{\mathrm{R}}\left(\tau_{2}\right)+\mathbf{n}_{\mathrm{R}, \mathrm{D}}\left(\tau_{2}\right) \\
& =\mathbf{h}_{\mathrm{R}, \mathrm{D}}^{a_{2}\left(\tau_{2}\right)} c_{m_{2}}^{\mathrm{R}}\left(\tau_{2}\right)+\mathbf{n}_{\mathrm{R}, \mathrm{D}}\left(\tau_{2}\right)
\end{aligned}
$$

where $\mathbf{H}_{\mathrm{S}, \mathrm{D}}$ and $\mathbf{H}_{\mathrm{R}, \mathrm{D}}$ are Rayleigh fading channel matrices defined like $\mathbf{H}_{\mathrm{S}, \mathrm{R}}$ in (6). $\mathbf{n}_{\mathrm{S}, \mathrm{D}}\left(\tau_{1}\right)$ and $\mathbf{n}_{\mathrm{R}, \mathrm{D}}\left(\tau_{2}\right)$ are additive Gaussian noise vectors, which are defined like $\mathbf{n}_{\mathrm{S}, \mathrm{R}}\left(\tau_{1}\right)$ in (6). Furthermore, $\mathbf{h}_{\mathrm{S}, \mathrm{D}}^{a_{1}\left(\tau_{1}\right)}$ and $\mathbf{h}_{\mathrm{R}, \mathrm{D}}^{a_{2}\left(\tau_{2}\right)}$ stand for the $a_{1}\left(\tau_{1}\right)$ th and $a_{2}\left(\tau_{2}\right)$ th columns of channel matrices $\mathbf{H}_{\mathrm{S}, \mathrm{D}}$ and $\mathbf{H}_{\mathrm{R}, \mathrm{D}}$, respectively. The SM demapper demodulates the received signal vectors $\mathbf{y}_{\mathrm{S}, \mathrm{D}}\left(\tau_{1}\right)$ and $\mathbf{y}_{\mathrm{R}, \mathrm{D}}\left(\tau_{2}\right)$ to generate the estimated binary codeword sequences $\hat{\mathbf{c}}_{1}$ and $\hat{\mathbf{c}}_{2}$, respectively. In the following, the 'bits to $M$-ary symbols' block maps $\hat{\mathbf{c}}_{1}$ and $\hat{\mathbf{c}}_{2}$ to the non-binary codeword sequences $\hat{\mathbf{v}}_{1}$ and $\hat{\mathbf{v}}_{2}$, respectively. Finally, the joint RS decoding which will be discussed in Sec. 5 is carried out to generate an estimate $\overline{\mathbf{m}}$ of binary information sequence $\mathbf{m}$.

\section{RS Coded SM (RSC-SM) Scheme}

This section presents the non-cooperative RSC-SM scheme as shown in Fig. 3. The proposed RSC-SM scheme also contains two different RS codes. Unlike the RSCCSM scheme, both RS codes $R S_{1}\left(N, K_{1}, d_{1}\right)$ and $R S_{2}\left(N, K_{2}, d_{2}\right)$ are placed at source node. The binary information sequence $\mathbf{m}$ is first mapped into non-binary $M$-ary symbol sequence $\mathbf{b}_{1}$ over $\operatorname{GF}\left(2^{n}\right)$ through the 'bits to $M$-ary symbols' block. We select the non-binary sequence $\mathbf{b}_{2}$ of length $K_{2}$ from the non-binary sequence $\mathbf{b}_{1}$ of length $K_{1}$ for partial encoding employing RS code $R S_{2}\left(N, K_{2}, d_{2}\right)$. The process is same as RSCC-SM scheme, explained in the previous section. The encoded non-binary codeword symbol sequences from two RS codes, i.e., $\mathbf{v}_{1}$ and $\mathbf{v}_{2}$ are multiplexed into a single nonbinary sequence $\mathbf{d}=\left[\mathbf{v}_{1}, \mathbf{v}_{2}\right]$. In the following, the binary sequence $\mathbf{c}$ is generated by the ' $M$-ary symbols to bits' block as shown in Fig. 3. After the SM mapper as discussed in Sec. 3, the transmission vector $\mathbf{c}_{m, a}(\tau)$ is generated as follows

$$
\mathbf{c}_{m, a}(\tau)=\left[\ldots, 0, c_{m}(\tau), 0, \ldots\right]^{\mathrm{T}}
$$

where $c_{m}(\tau)$ is the $L$-QAM/PSK symbol transmitted at the $a(\tau)$ th antenna, $m \in\{1,2, \ldots, L\}, a(\tau) \in\left\{1,2, \ldots, N_{\mathrm{T}}\right\}$ and $\tau \in\{1,2, \ldots, 2 N\}$. At the destination node, the received vector $\mathbf{y}(\tau)$ is written as

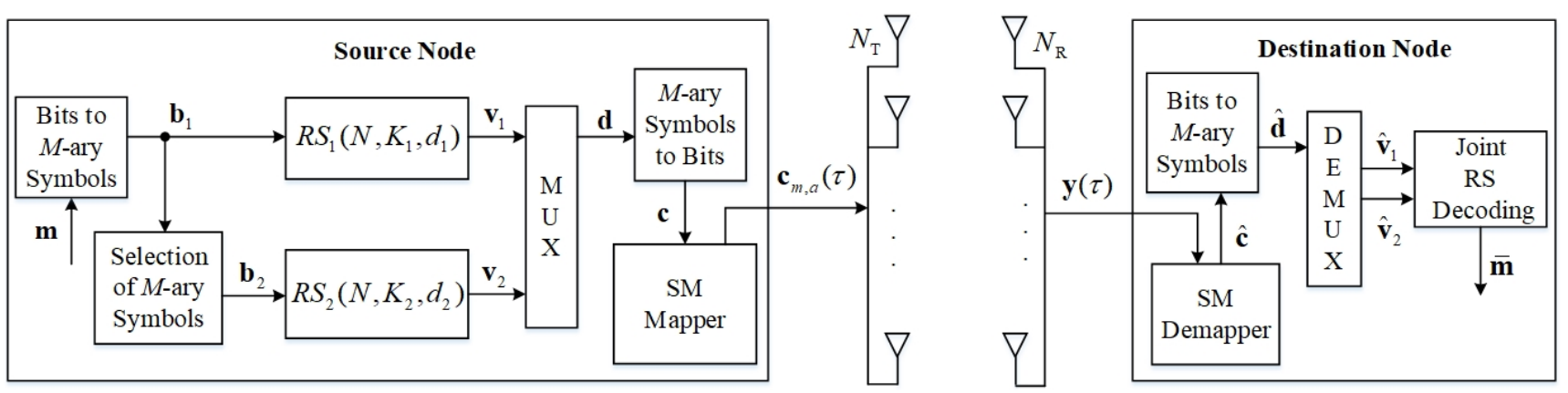

Fig. 3. Non-cooperative RSC-SM scheme. 


$$
\begin{aligned}
\mathbf{y}(\tau) & =\mathbf{H} \mathbf{c}_{m, a}(\tau)+\mathbf{n}(\tau) \\
& =\mathbf{h}^{a(\tau)} c_{m}(\tau)+\mathbf{n}(\tau)
\end{aligned}
$$

where $\mathbf{H}$ denotes the $N_{\mathrm{R}} \times N_{\mathrm{T}}$ quasi-static Rayleigh fading channel matrix defined like $\mathbf{H}_{\mathrm{S}, \mathrm{R}}$ in (6), and $\mathbf{h}^{a(\tau)}$ is the $a(\tau)$ th column vector of the channel matrix $\mathbf{H}$. Furthermore, $\mathbf{n}(\tau)$ represents the $N_{\mathrm{R}} \times 1$ additive Gaussian noise vector defined similar to $\mathbf{n}_{\mathrm{S}, \mathrm{R}}\left(\tau_{1}\right)$ in (6). The $\mathrm{SM}$ demapper at the destination carries out the demodulation for the received signal. The estimated binary sequence $\hat{\mathbf{c}}$ is then generated. After that, $\hat{\mathbf{c}}$ is transformed into $M$-ary non-binary sequence $\hat{\mathbf{d}}$. Through the de-multiplexer, two non-binary coded sequences $\hat{\mathbf{v}}_{1}$ and $\hat{\mathbf{v}}_{2}$ are obtained. Finally, the joint RS decoding is performed to get the estimated binary information sequence $\overline{\mathbf{m}}$.

\section{Joint RS Decoding}

Joint RS decoding is a key feature of the proposed RSCC-SM scheme and RSC-SM scheme. In this section, we propose two joint decoding schemes, i.e., naive approach and smart approach. The detailed contents will be shown in the following two subsections.

\subsection{Naive Approach}

The naive approach for joint RS decoding is shown in Fig. 4. The $M$-ary $R S_{1}$ and $R S_{2}$ decoders that employ Euclidean decoding algorithm decode the non-binary codeword sequences $\hat{\mathbf{v}}_{1}$ and $\hat{\mathbf{v}}_{2}$, respectively. The decoded output non-binary information sequences $\hat{\mathbf{b}}_{1}$ and $\hat{\mathbf{b}}_{2}$ are given into the combiner block during their respective time slots. It should be noted that all $K_{2}$ symbols of $\hat{\mathbf{b}}_{2}$ are more reliable than the first $K_{2}$ symbols of $\hat{\mathbf{b}}_{1}$ for a high signalto-noise ratio (SNR) due to the larger minimum distance $d_{2}$ of RS code $R S_{2}$ as compared to $R S_{1}$. In channel coding, the BER performance of code with more error correcting capability is worse than the code with less error correcting capability for low SNR regime while it outperforms the

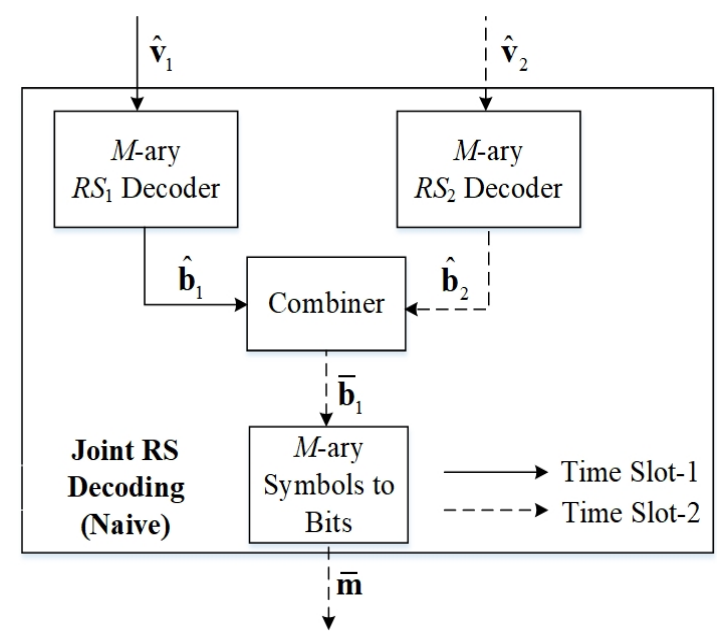

Fig. 4. Joint RS decoding (naive approach). less error correcting capability code over high SNR. Therefore, we set a threshold $\sigma$, which is taken as the SNR where the BER performance (individual performance) of $R S_{1}$ and $R S_{2}$ codes crosses each other or meets each other. The threshold value can be obtained by pre-simulating the two signals. In the case of $\mathrm{SNR} \leq \sigma$, the output of the combiner is $\overline{\mathbf{b}}_{1}=\hat{\mathbf{b}}_{1}$. When $\mathrm{SNR}>\sigma$, the first $K_{2}$ symbols of sequence $\hat{\mathbf{b}}_{1}$ are replaced by sequence $\hat{\mathbf{b}}_{2}$, and the combiner block outputs $\overline{\mathbf{b}}_{1}=\left[\hat{\mathbf{b}}_{2}, \hat{\mathbf{b}}_{1}^{\prime}\right]$, where $\hat{\mathbf{b}}_{1}^{\prime}=\left[\hat{b}_{K_{2}}^{(1)}, \hat{b}_{K_{2}+1}^{(1)}, \ldots, \hat{b}_{K_{2}+\left(K_{1}-K_{2}\right)-1}^{(1)}\right]$. Finally, the estimated binary information sequence $\overline{\mathbf{m}}$ is yielded through the ' $M$ ary symbols to bits' block.

\subsection{Smart Approach}

In this subsection, we propose another approach for joint RS decoding i.e., smart approach. Figure 5 shows the illustrative diagram of smart approach, which is a serial decoding that effectively replaces some source transmitted symbols with more useful information symbols communicated by the relay. Firstly, the non-binary codeword sequence $\hat{\mathbf{v}}_{2}$ is given into the $M$-ary $R S_{2}$ decoder to generate an estimated information sequence $\hat{\mathbf{b}}_{2}$ of sequence $\mathbf{b}_{2}$. Since the demodulated codeword sequence $\hat{\mathbf{v}}_{1}$ contains the parity symbol sequence $\hat{\mathbf{p}}_{1}$ and information symbol sequence $\hat{\mathbf{b}}_{1}$, therefore the first $K_{2}$ symbols of $\hat{\mathbf{b}}_{1}$ are replaced by $\hat{\mathbf{b}}_{2}$. After the combiner block, the non-binary sequence $\overline{\mathbf{v}}_{1}=\left[\hat{\mathbf{p}}_{1}, \hat{\mathbf{b}}_{2}, \hat{\mathbf{b}}_{1}^{\prime}\right]$ of length $N$ is generated. This joint source relay sequence $\overline{\mathbf{v}}_{1}$ has more reliability and possesses greater error correcting capability than $\hat{\mathbf{v}}_{1}$ due to the inclusion of sequence $\hat{\mathbf{b}}_{2}$. This action provides more coding gain to the coded cooperative scheme. Then, the non-binary sequence $\overline{\mathbf{v}}_{1}$ is fed to the $M$-ary $R S_{1}$ decoder that produces an estimate $\overline{\mathbf{b}}_{1}$ of information symbol sequence $\mathbf{b}_{1}$. Finally, the non-binary sequence $\overline{\mathbf{b}}_{1}$ is similarly transformed into binary information sequence $\overline{\mathbf{m}}$ as the estimation of information sequence $\mathbf{m}$ like naive approach. As compared to the naive approach, the smart approach will obtain an added advantage since more reliable decoded symbols of $R S_{2}$ decoder are utilized as an input to $R S_{1}$ decoder.

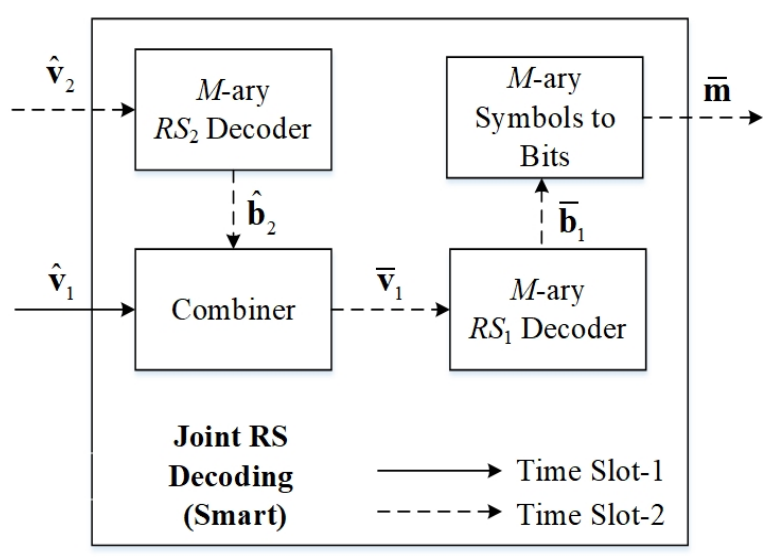

Fig. 5. Joint RS decoding (smart approach). 


\section{Simulation Results}

This section presents the BER performance of proposed RSCC-SM and RSC-SM schemes through quasistatic Rayleigh fading channel. The number of antennas at the source node and the relay node are kept four. However, at the destination node, the number of antennas $\left(N_{\mathrm{R}}\right)$ may vary in some situations. Two different scenarios have been considered to generalize our proposed RSCC-SM and RSC-SM schemes. For the first scenario, we employ RS codes $R S_{1}(63,51,13)$ and $R S_{2}(63,31,33)$ over the field $\mathrm{GF}\left(2^{6}\right)$ constructed based on the polynomial $1+X+X^{6}$. In this scenario, their code rates are $R_{1}=51 / 63$ and $R_{2}=31 / 63$ for $R S_{1}(63,51,13)$ and $R S_{2}(63,31,33)$, respectively. Furthermore, the RS codes $R S_{1}(255,239,17)$ and $R S_{2}(255,128,128)$ over $\mathrm{GF}\left(2^{8}\right)$ which is constructed using polynomial $1+X+X^{8}$ are considered in the second scenario. Their code rates are $R_{1}=239 / 255$ and $R_{1}=128 / 255$, respectively. For the first scenario, 16- QAM and $N_{\mathrm{T}}=4$ are employed. However, we employ 64-QAM and $N_{\mathrm{T}}=4$ for the second scenario. It is assumed that relay node has a $2 \mathrm{~dB}$ SNR gain over source node, i.e., $\lambda_{\mathrm{R}, \mathrm{D}}=\lambda_{\mathrm{S}, \mathrm{D}}+2$. All the simulations are reported in terms of $\mathrm{E}_{\mathrm{b}} / \mathrm{N}_{0}$ versus BER, where $E_{b} / N_{0}$ refers to the SNR per bit of the direct link i.e., source-to-destination link. Moreover, Euclidean decoding algorithm is utilized in all simulations.

\subsection{Performance Comparison of RSCC-SM Scheme Using Different Ways of Information Selection}

Figure 6 presents the BER performance comparison of RSCC-SM scheme using different ways of information selection at the relay node for the first scenario. The infor-

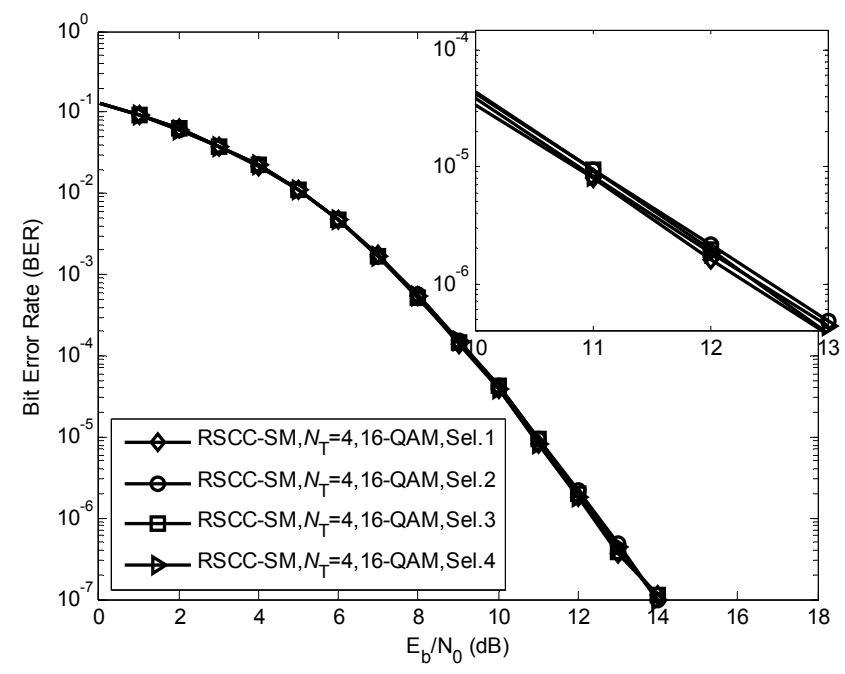

Fig. 6. BER performance comparison of RSCC-SM scheme (16-QAM) with $R S_{1}(63,51,13)$ and $R S_{2}(63,31,33)$ under different ways of information selection, $N_{\mathrm{T}}=N_{\mathrm{R}}=4$. The main figure shows the BER performance in the SNR range of $0-14 \mathrm{~dB}$, whereas the figure in the top right corner shows the BER performance in the SNR range of 10-13 dB.

\begin{tabular}{|c|c|}
\hline Serial number & Information selection patterns \\
\hline Sel. 1 & 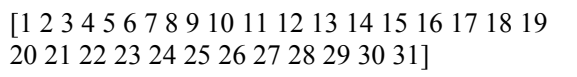 \\
\hline Sel. 2 & 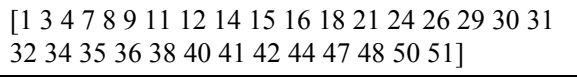 \\
\hline Sel. 3 & 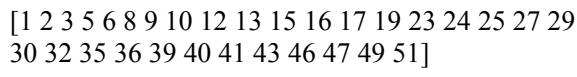 \\
\hline Sel. 4 & 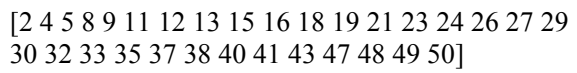 \\
\hline
\end{tabular}

Tab. 3. Information selection patterns for the first scenario.

mation selection patterns are randomly generated. The ideal source to relay channel $\left(\lambda_{\mathrm{S}, \mathrm{R}}=\propto\right)$, joint RS decoding (smart approach) and $N_{\mathrm{R}}=4$ receive antennas are used. Table 3 lists various information selection patterns in terms of the first scenario. Each information selection pattern is a vector of length $K_{2}$, where every element denotes the position of the selected symbol in the estimated information symbol sequence of RS code $R S_{1}$. The simulation results in Fig. 6 reveal that the BER performance of RSCCSM scheme under different information selection methods almost overlaps each other over the whole SNR range. For convenience, we select the first 31 symbols from the estimated information sequence in the first scenario. Similarly, the first 128 symbols are selected from the estimated information sequence in the second scenario.

\subsection{Performance of RSCC-SM Scheme under Non-ideal and Ideal Source to Relay Channels and RSC-SM Scheme}

The BER performance of RSCC-SM and RSC-SM schemes for the first scenario is exhibited in Fig. 7. The joint decoding (smart approach) is employed. We observe that the RSCC-SM scheme over both non-ideal (practical) source to relay channel $\left(\lambda_{\mathrm{S}, \mathrm{R}} \neq \propto\right)$ and ideal source to relay channel $\left(\lambda_{\mathrm{S}, \mathrm{R}}=\propto\right)$ outperforms the RSC-SM scheme. In Fig. 7, the RSCC-SM scheme for the cases of $\lambda_{\mathrm{S}, \mathrm{R}}=8 \mathrm{~dB}$ and $\lambda_{\mathrm{S}, \mathrm{R}}=\propto$ gets a gain of about $1 \mathrm{~dB}$ and $1.1 \mathrm{~dB}$ over RSC-SM at BER $=5 \times 10^{-7}$, respectively. This is because the cooperation between source and relay nodes makes the coded cooperative SM scheme get an evident performance gain. Furthermore, the RSCC-SM scheme under $\lambda_{\mathrm{S}, \mathrm{R}}=$ $8 \mathrm{~dB}$ only lags behind by about $0.1 \mathrm{~dB}$ gain as compared to its counterpart RSCC-SM scheme under $\lambda_{\mathrm{S}, \mathrm{R}}=\propto$. This shows that the usefulness of RSCC-SM system in the practical scenario. Moreover, Figure 8 analyzes the BER performance of RSCC-SM scheme under $\lambda_{\mathrm{S}, \mathrm{R}}=14 \mathrm{~dB}$ and $\lambda_{\mathrm{S}, \mathrm{R}}=\propto$ and RSC-SM scheme for the second scenario. The smart decoding approach is adopted. At BER $=3 \times 10^{-6}$, the RSCC-SM scheme under the conditions of $\lambda_{\mathrm{S}, \mathrm{R}}=14 \mathrm{~dB}$ and $\lambda_{\mathrm{S}, \mathrm{R}}=\propto$ separately achieves about $1 \mathrm{~dB}$ and $1.1 \mathrm{~dB}$ gains over the RSC-SM scheme. Similarly, the RSCC-SM scheme in the case of $\lambda_{\mathrm{S}, \mathrm{R}}=14 \mathrm{~dB}$ only lags behind by about $0.1 \mathrm{~dB}$ gain over the RSCC-SM scheme in the case of $\lambda_{\mathrm{S}, \mathrm{R}}=\propto$. 


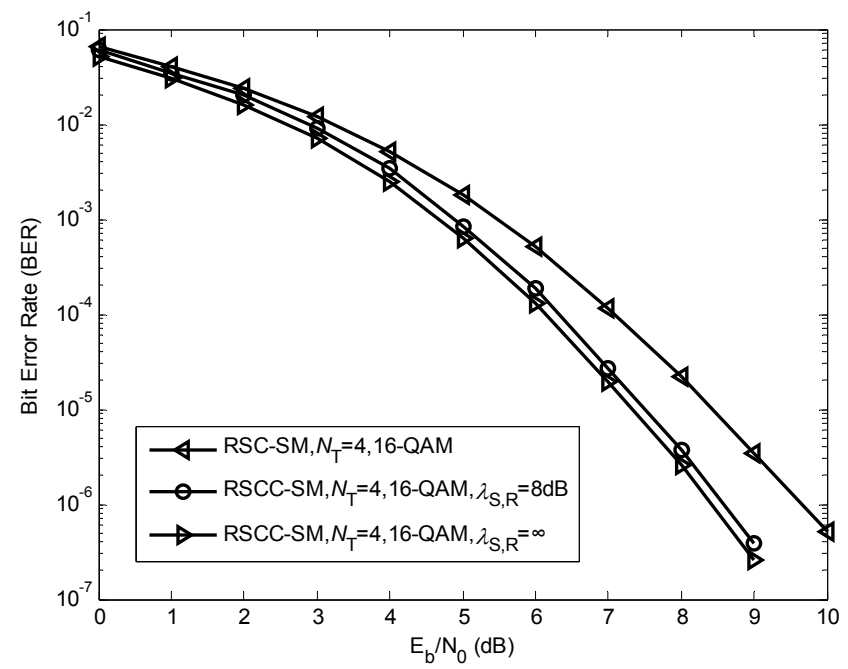

Fig. 7. BER performance of RSCC-SM scheme under nonideal and ideal source to relay channels and RSC-SM scheme (16-QAM) employing $R S_{1}(63,51,13)$ and $R S_{2}(63,31,33), N_{\mathrm{T}}=4, N_{\mathrm{R}}=6$.

Furthermore, Figure 9 shows the BER performance of the RSCC-SM scheme with $\lambda_{\mathrm{S}, \mathrm{R}}=\lambda_{\mathrm{S}, \mathrm{D}}, \lambda_{\mathrm{S}, \mathrm{R}}=13 \mathrm{~dB}$ and $\lambda_{\mathrm{S}, \mathrm{R}}=\propto$ for the first scenario. The smart decoding approach is utilized in our simulations. Monte Carlo simulations reveal that the RSCC-SM scheme with $\lambda_{\mathrm{S}, \mathrm{R}}=\lambda_{\mathrm{S}, \mathrm{D}}$ performs worse BER performance than its counterpart RSCC-SM scheme with $\lambda_{\mathrm{S}, \mathrm{R}}=13 \mathrm{~dB}$ and $\lambda_{\mathrm{S}, \mathrm{R}}=\propto$ under identical conditions. As shown in Fig. 9, the RSCC-SM scheme with $\lambda_{\mathrm{S}, \mathrm{R}}=\lambda_{\mathrm{S}, \mathrm{D}}$ has a loss in SNR of approximately $0.5 \mathrm{~dB}$ and $0.7 \mathrm{~dB}$ over the RSCC-SM scheme with $\lambda_{\mathrm{S}, \mathrm{R}}=13 \mathrm{~dB}$ and $\lambda_{\mathrm{S}, \mathrm{R}}=\propto$, respectively, at a BER of $10^{-5}$. Furthermore, the BER performance gap of the RSCC-SM scheme between $\lambda_{\mathrm{S}, \mathrm{R}}=\lambda_{\mathrm{S}, \mathrm{D}}$ and $\lambda_{\mathrm{S}, \mathrm{R}}=13 \mathrm{~dB}$ and $\lambda_{\mathrm{S}, \mathrm{R}}=\propto$, respectively, is large at low SNR. However, the performance gap is further reduced at high SNR.

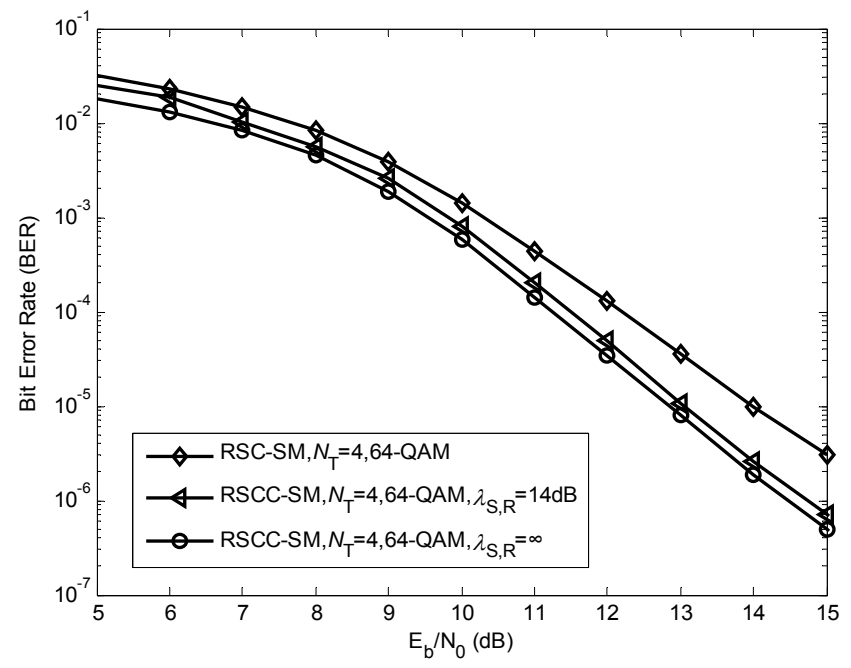

Fig. 8. BER performance of RSCC-SM scheme under nonideal and ideal source to relay channels and RSC-SM scheme (64-QAM) employing $R S_{1}(255,239,17)$ and $R S_{2}(255,128,128), N_{\mathrm{T}}=4, N_{\mathrm{R}}=6$.

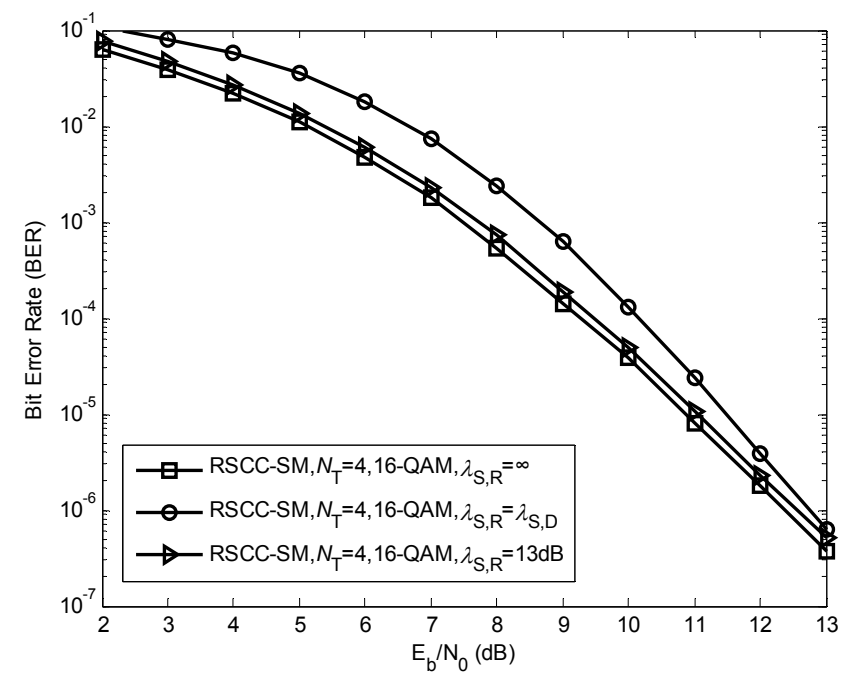

Fig. 9. BER performance of RSCC-SM scheme (16-QAM) employing $R S_{1}(63,51,13)$ and $R S_{2}(63,31,33)$, with $\lambda_{\mathrm{S}, \mathrm{R}}=\lambda_{\mathrm{S}, \mathrm{D}}, \lambda_{\mathrm{S}, \mathrm{R}}=13 \mathrm{~dB}$ and $\lambda_{\mathrm{S}, \mathrm{R}}=\propto, N_{\mathrm{T}}=N_{\mathrm{R}}=4$.

\subsection{Performance Comparison of RSCC-SM and RSC-SM Schemes under Naive and Smart Approaches}

Figure 10 demonstrates the BER performance comparison of RSCC-SM scheme with joint decoding (naive and smart approaches) for the first scenario under different number of receive antennas. The ideal source to relay link $\left(\lambda_{\mathrm{S}, \mathrm{R}}=\propto\right)$ is supposed. At BER $=6.7 \times 10^{-5}$, the RSCC-SM scheme utilizing smart approach obtains around $1.3 \mathrm{~dB}$ SNR over its counterpart RSCC-SM scheme using naive approach under $N_{\mathrm{R}}=4$. In the case of $N_{\mathrm{R}}=4$, the gain of RSCC-SM scheme (smart approach) as compared with its counterpart scheme (naive approach) is about $1.8 \mathrm{~dB}$ at $\mathrm{BER}=2 \times 10^{-7}$.

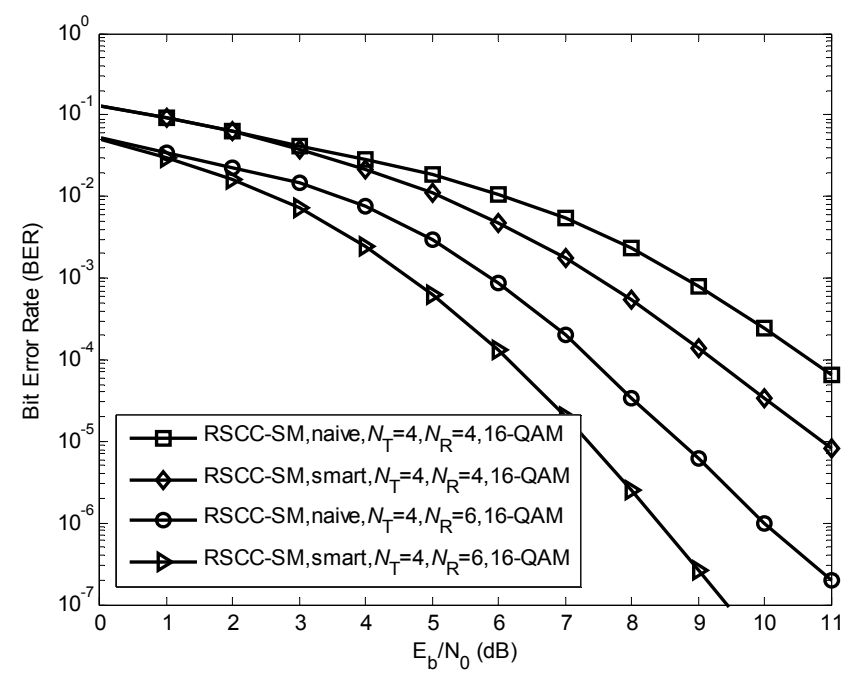

Fig. 10. BER performance comparison of RSCC-SM scheme (16-QAM) with $R S_{1}(63,51,13)$ and $R S_{2}(63,31,33)$ employing joint RS decoding (naive and smart approaches), $N_{\mathrm{T}}=4$. 


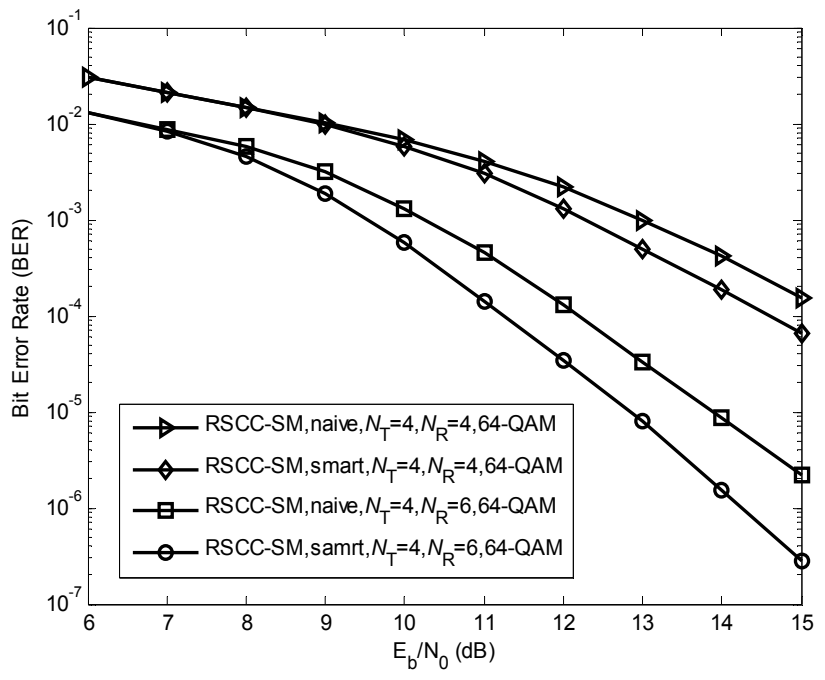

Fig. 11. BER performance comparison of RSCC-SM scheme (64-QAM) with $R S_{1}(255,239,17)$ and $R S_{2}(255,128,128)$ employing joint RS decoding (naive and smart approaches), $N_{\mathrm{T}}=4$.

Similarly, Figure 11 investigates the BER performance of RSCC-SM scheme (naive and smart approaches) for the second scenario with different number of receive antennas. We assume that the ideal source to relay link $\left(\lambda_{\mathrm{S}, \mathrm{R}}=\propto\right)$ is adopted in the simulation. For $N_{\mathrm{R}}=4$ and 6 , the RSCC-SM scheme with smart approach separately outperforms that of naive approach by about $0.8 \mathrm{~dB}$ and $1 \mathrm{~dB}$ gains at $\mathrm{BER}=1.5 \times 10^{-4}$. Figures 12 and 13 show the BER performance of the non-cooperative RSC-SM scheme with joint RS decoding (naive and smart approaches) for the two scenarios under various number of receive antennas, respectively. The simulated results confirm the fact that the scheme employing smart approach achieves better BER performance than that of naive approach under identical conditions once again.

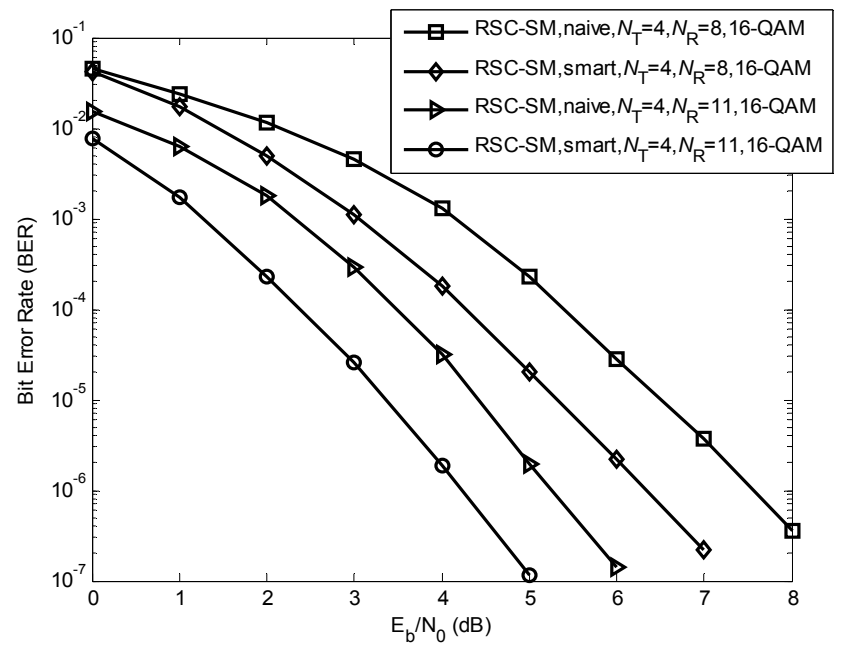

Fig. 12. BER performance of non-cooperative RSC-SM scheme (16-QAM) with $R S_{1}(63,51,13)$ and $R S_{2}(63,31,33)$ employing joint RS decoding (naive and smart approaches), $N_{\mathrm{T}}=4$.

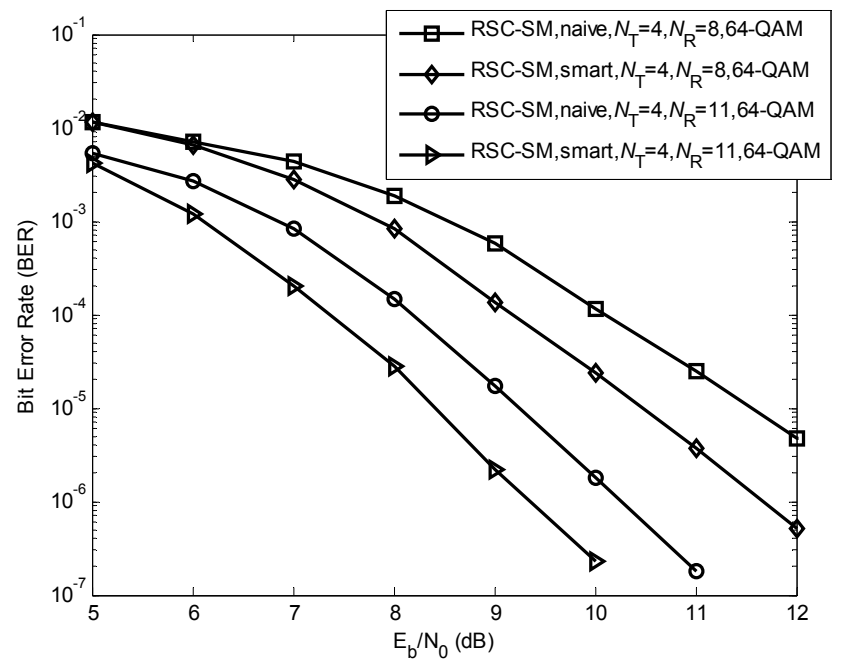

Fig. 13. BER performance of non-cooperative RSC-SM scheme (64-QAM) with $R S_{1}(255,239,17)$ and $R S_{2}(255,128,128)$ employing joint $\mathrm{RS}$ decoding (naive and smart approaches), $N_{\mathrm{T}}=4$.

\subsection{Performance Comparison of RSCC-SM Scheme under Various Number of Receive Antennas}

Figures 14 and 15 discuss the BER performance of RSCC-SM scheme with various number of receive antennas under ideal source to relay link $\left(\lambda_{\mathrm{S}, \mathrm{R}}=\propto\right)$ for our considered two scenarios. In the simulation, the joint decoding (smart approach) is used. Monte Carlo simulations present that the BER performance of coded cooperative SM scheme is enhanced as the number of receive antenna increases. In Fig. 14, the RSCC-SM scheme having $N_{\mathrm{R}}=3$ receive antennas achieves a BER of $2.4 \times 10^{-3}$ at an SNR of $9 \mathrm{~dB}$. Under $N_{\mathrm{R}}=4,5$ and 6 , the corresponding BER performances i.e., $1.4 \times 10^{-4}, 6.6 \times 10^{-6}$ and $2.6 \times 10^{-7}$ are

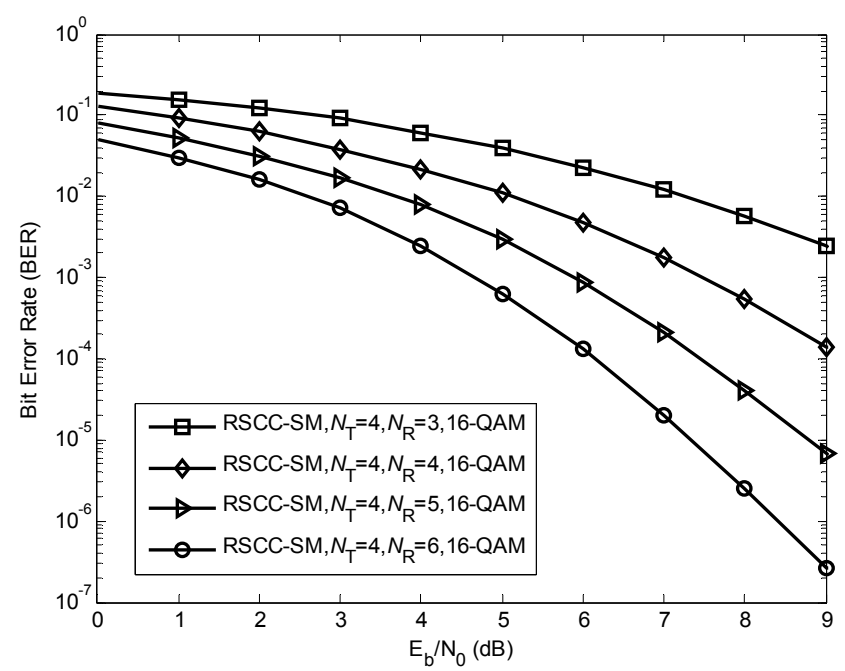

Fig. 14. BER performance of RSCC-SM scheme (16-QAM) with $R S_{1}(63,51,13)$ and $R S_{2}(63,31,33)$ under various number of receive antennas, $N_{\mathrm{T}}=4$ 


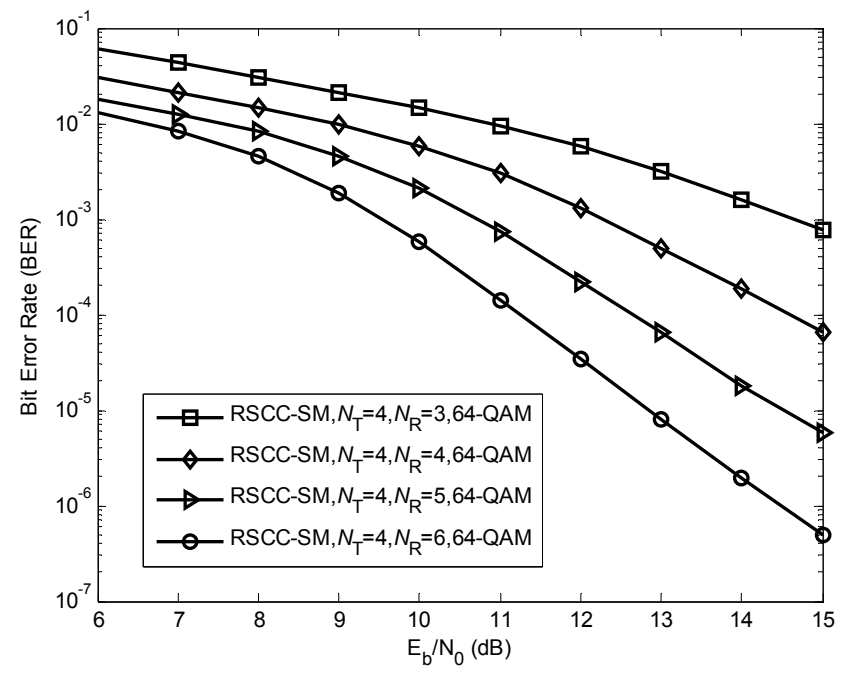

Fig. 15. BER performance of RSCC-SM scheme (64-QAM) with $R S_{1}(255,239,17)$ and $R S_{2}(255,128,128)$ under various number of receive antennas, $N_{\mathrm{T}}=4$.

obtained at the same SNR. Furthermore, the RSCC-SM scheme with $N_{\mathrm{R}}=3,4,5$ and 6 receive antennas achieves a BER of $7.7 \times 10^{-4}, 6.5 \times 10^{-5}, 5.8 \times 10^{-6}$ and $4.8 \times 10^{-7}$ at $\mathrm{SNR}=15 \mathrm{~dB}$, respectively, as shown in Fig. 15 .

\subsection{Performance Comparison of RSC-SM and RSCC-SM Schemes with the Existed Schemes}

The BER performance comparison of the RSC-SM and RSCC-SM schemes employing smart approach for the first scenario with the existed schemes in [16] i.e., polar coded SM (PC-SM), multi-level polar coded SM (MLPC$\mathrm{SM})$ and multi-level polar coded cooperative SM (MLPCC-SM) schemes is presented in Fig. 16. The PC-SM and MLPC-SM schemes are served as the benchmark to the RSC-SM scheme. Similarly, the MLPCC-SM scheme is used as a benchmark to the RSCC-SM scheme. It can be seen from Fig. 16 that our proposed RSC-SM scheme outperforms the PC-SM and MLPC-SM schemes under identical conditions such as 16-QAM, $N_{\mathrm{T}}=4$ and $N_{\mathrm{R}}=4$. For example, the RSC-SM scheme obtains respective gains of about $5.2 \mathrm{~dB}$ and $4.7 \mathrm{~dB}$ at a BER of $4.9 \times 10^{-4}$ as compared to the PC-SM and MLPC-SM schemes. Furthermore, Monte Carlo simulations also show that our proposed RSCC-SM scheme achieves a better BER performance over its counterpart MLPCC-SM scheme under identical conditions such as $\lambda_{\mathrm{S}, \mathrm{R}}=\propto, 16-\mathrm{QAM}, N_{\mathrm{T}}=4$ and $N_{\mathrm{R}}=4$ as shown in Fig. 16.

\subsection{Comparison of Theoretical Curves with Simulation Results for RSCC-SM Scheme}

This section discusses the theoretical and simulated BER of the RSCC-SM scheme employing smart decoding approach under $\lambda_{\mathrm{S}, \mathrm{R}}=\propto$ for the first and second scenarios. For the $t_{1}$-error-correcting $\mathrm{RS}$ code $R S_{1}\left(N, K_{1}, d_{1}\right)$ in source

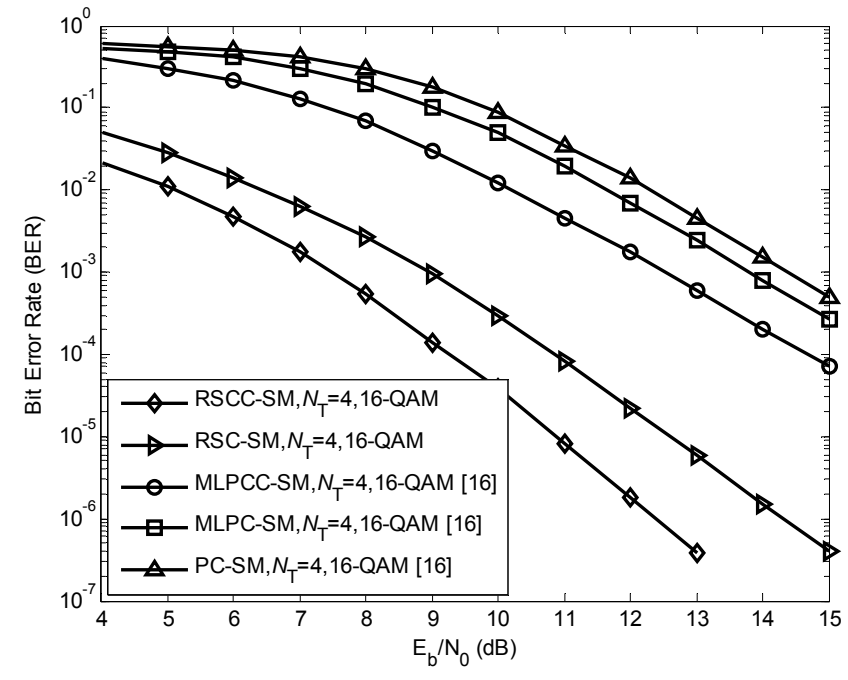

Fig. 16. BER performance for RSC-SM and RSCC-SM schemes (16-QAM) with $R S_{1}(63,51,13)$ and $R S_{2}(63,31,33)$, PC-SM [16], MLPC-SM [16] and MLPCC-SM [16] schemes, $N_{\mathrm{T}}=N_{\mathrm{R}}=4$.

to destination channel, the symbol error rate (SER) is expressed as [17]

$$
P_{\mathrm{e}}^{(1)} \approx \frac{1}{N} \sum_{\mu_{1}=t_{1}+1}^{N} \mu_{1}\left(\begin{array}{l}
N \\
\mu_{1}
\end{array}\right)\left(p^{(1)}\right)^{\mu_{1}}\left(1-p^{(1)}\right)^{N-\mu_{1}}
$$

where $\left(\begin{array}{l}N \\ \mu_{1}\end{array}\right)$ denotes the binomial coefficient and $p^{(1)}$ represents the symbol error probabilities of SM. Based on the introduction in [3], the parameter $p^{(1)}$ can be written as

$$
p^{(1)}=p_{\mathrm{a}}^{(1)}+p_{\mathrm{d}}^{(1)}-p_{\mathrm{a}}^{(1)} p_{\mathrm{d}}^{(1)}
$$

where $p_{\mathrm{a}}{ }^{(1)}$ and $p_{\mathrm{d}}{ }^{(1)}$ denote the error estimated probabilities of antenna index and modulated symbol, respectively. The detailed derivation of $p_{\mathrm{a}}{ }^{(1)}$ and $p_{\mathrm{d}}{ }^{(1)}$ is presented in [3], which shows that they are related to the SNR of source to destination link.

Similarly, the SER of $t_{2}$-error-correcting RS code $R S_{2}\left(N, K_{2}, d_{2}\right)$ under relay to destination channel is given by [17]

$$
P_{\mathrm{e}}^{(2)} \approx \frac{1}{N} \sum_{\mu_{2}=t_{2}+1}^{N} \mu_{2}\left(\begin{array}{l}
N \\
\mu_{2}
\end{array}\right)\left(p^{(2)}\right)^{\mu_{2}}\left(1-p^{(2)}\right)^{N-\mu_{2}}
$$

where $p^{(2)}$ is defined like $p^{(1)}$ in (15).

Based on the analysis and introduction in [17], the approximate BER bound of our proposed RSCC-SM scheme employing smart decoding approach can be denoted as

$$
P_{\mathrm{e}} \approx \frac{1}{\log _{2}^{N+1}}\left(\frac{K_{1}-K_{2}}{K_{1}} P_{\mathrm{e}}^{(1)}+\frac{K_{2}}{K_{1}} P_{\mathrm{e}}^{(2)}\right) .
$$

From the simulation results of Figs. 17 and 18, we clearly observe that the theoretical bound and simulation results show a close match at their high SNR region, which validates the analytical frameworks. 


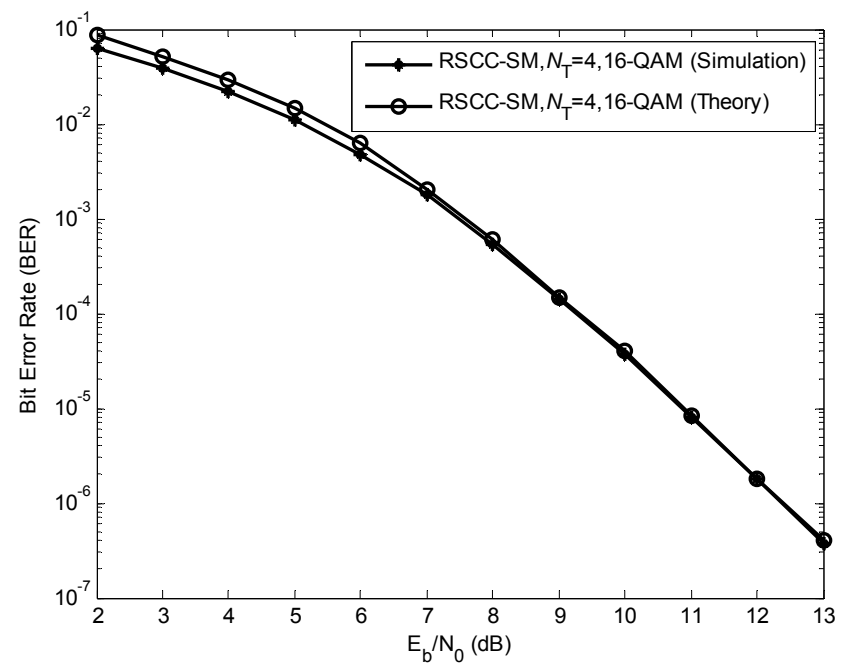

Fig. 17. Comparison between theoretical and simulated BER of the RSCC-SM scheme (16-QAM) with $R S_{1}(63,51,13)$ and $R S_{2}(63,31,33), N_{\mathrm{T}}=N_{\mathrm{R}}=4$.

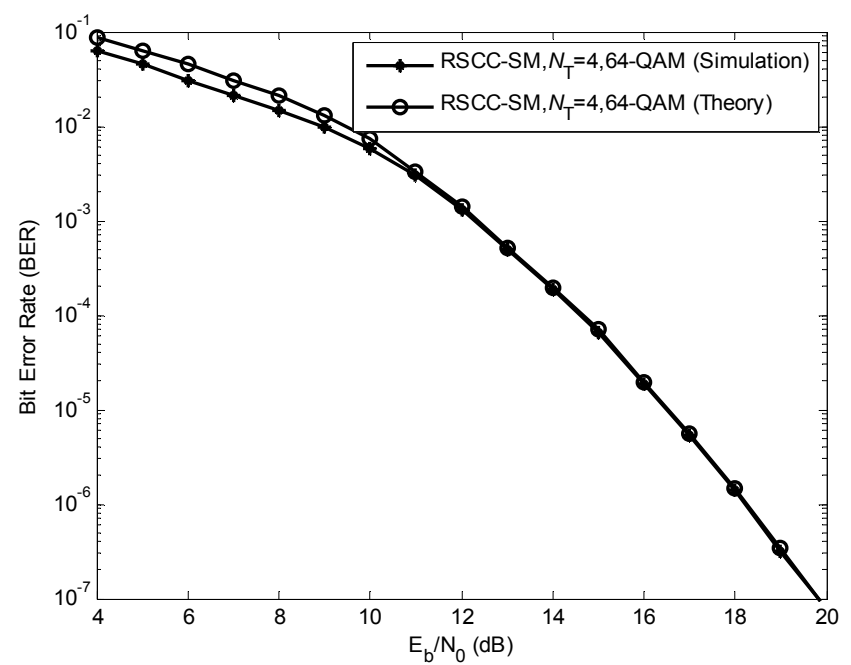

Fig. 18. Comparison between theoretical and simulated BER of the RSCC-SM scheme (64-QAM) with $R S_{1}(255,239,17)$ and $R S_{2}(255,128,128), N_{\mathrm{T}}=N_{\mathrm{R}}=4$.

\subsection{Performance of RSC-SM Scheme over Uncorrelated and Correlated Quasi-static Rayleigh Fading Channels}

In this subsection, we discuss the BER performance of RSC-SM scheme over uncorrelated and correlated Rayleigh fading channels. The considered correlated channel matrix is modeled by $\mathbf{H}_{\text {corr }}=\mathbf{Z}_{\eta}^{1 / 2} \mathbf{H} \mathbf{Z}_{\mathrm{r}}^{1 / 2}$, where $\mathbf{Z}_{\eta}=\left[z_{p q}\right]_{N_{\mathrm{T}} \times N_{\mathrm{T}}}$ and $\mathbf{Z}_{\mathrm{r}}=\left[z_{p q}\right]_{N_{\mathrm{R}} \times N_{\mathrm{R}}}$ are the spatial correlation matrices at the transmitter and the receiver, respectively $[3,18]$. In the simulation, the exponential correlation matrix model is assumed in which $z_{p q}=z_{q p}^{*}=z^{|q-p|}$ and $|z|<1$ with $z$ being the amount of correlation. Figure 19 shows the BER curves of RSC-SM scheme employing smart decoding approach for the first scenario over uncorrelated $(z=0)$ and correlated $(z=0.5$ and 0.8$)$ channels in

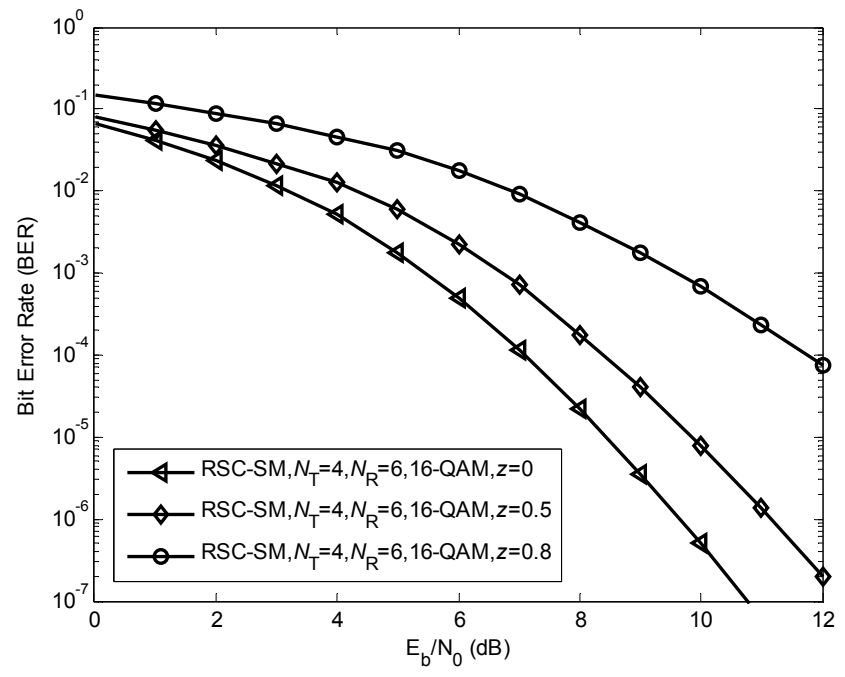

Fig. 19. BER performance of RSC-SM scheme (16-QAM) with $R S_{1}(63,51,13)$ and $R S_{2}(63,31,33)$ over uncorrelated and correlated channels, $N_{\mathrm{T}}=4, N_{\mathrm{R}}=6$.

a $4 \times 6$ MIMO. The simulated results reveal that the BER performance of the RSC-SM scheme under correlated $(z=0.5$ and 0.8$)$ channel is degraded when compared with its counterpart RSC-SM scheme under uncorrelated $(z=0)$ channel. This implies the correlated channel is the best fit for the realistic scenarios.

\section{Conclusion}

The RSCC-SM scheme over quasi-static Rayleigh fading channel is proposed in this paper. In the proposed RSCC-SM scheme, two different RS codes with less and larger number of consecutive roots are employed at the source and relay nodes, respectively. The larger number of consecutive roots provides extra redundancy at the relay node. Therefore, some of non-binary information symbols are retransmitted by the relay node after partial encoding of source information symbols. To decode the received sequences at the common destination node, a joint RS decoder based on two different approaches, i.e., naive approach and smart approach are proposed. The simulated results demonstrate that the prominence of the proposed RSCC-SM scheme employing joint decoding (smart approach) over the non-cooperative RSC-SM scheme as well as the RSCC-SM scheme (naive approach) under identical conditions. Furthermore, we present the BER performance of the RSC-SM and RSCC-SM schemes under other conditions.

\section{Acknowledgments}

The authors would like to thank the Editor and anonymous reviewers for their precious suggestions, comments and reviews that help in improving the quality of this paper. 


\section{References}

[1] GUO, S., ZHANG, H. X., ZHANG, P, et al. Signal shaping for generalized spatial modulation and generalized quadrature spatial modulation. IEEE Transactions on Wireless Communications, 2019, vol. 18, no. 8, p. 4047-4059. DOI: 10.1109/TWC.2019.2920822

[2] ZHAO, C., YANG, F., UMAR, R., et al. Two-source asymmetric turbo-coded cooperative spatial modulation scheme with code matched interleaver. Electronics, 2020, vol. 9, no. 1, p. 1-20. DOI: 10.3390/electronics9010169

[3] MESLEH, R. Y., HAAS, H., SINANOVIC, S. Spatial modulation. IEEE Transactions on Vehicular Technology, 2008, vol. 57, no. 4, p. 2228-2241. DOI: 10.1109/TVT.2008.912136

[4] FENG, D., XU, H., ZHENG, J., et al. Nonbinary LDPC-coded spatial modulation. IEEE Transactions on Wireless Communications, 2018, vol. 17, no. 4, p. 2786-2799. DOI: 10.1109/TWC.2018.2803170

[5] VAN DER MEULEN, E. C. Three-terminal communication channels. Advances in Applied Probability, 1971, vol. 3, no. 1, p. 120-154. DOI: $10.2307 / 1426331$

[6] MESLEH, R. Y., IKKI, S. S. Performance analysis of spatial modulation with multiple decode and forward relays. IEEE Wireless Communications Letters, 2013, vol. 2, no. 4, p. 423-426. DOI: 10.1109/WCL.2013.051513.130256

[7] EJAZ, S., YANG, F., XU, H. Labeling diversity for $2 \times 2$ WLAN coded-cooperative networks. RadioEngineering, 2015, vol. 24, no. 2 , p. 470-480. DOI: 10.13164/re.2015.0470

[8] HUNTER, T. E., NOSRATINIA, A. Diversity through coded cooperation. IEEE Transactions on Wireless Communications, 2006, vol. 5, no. 2, p. 283-289. DOI: 10.1109/TWC.2006.02006

[9] ZHANG, S. W., YANG, F. F., TANG, L., et al. Joint design of QC-LDPC codes for coded cooperation system with joint iterative decoding. International Journal of Electronics, 2015, vol. 103, no. 3, p. 384-405. DOI: 10.1080/00207217.2015.1036374

[10] MUGHAL, S., YANG, F. F., EJAZ, S., et al. Asymmetric turbo code for coded-cooperative wireless communication based on matched interleaver with channel estimation and multi-receive antennas at the destination. RadioEngineering, 2017, vol. 26, no. 3, p. $878-889$. DOI: $10.13164 /$ re. 2017.0878

[11] UMAR, R., YANG, F. F., XU, H. J., et al. Multi-level construction of polar coded single carrier-FDMA based on MIMO antennas for coded cooperative wireless communication. IET Communications, 2018, vol. 12 , no. 10 , p. $1253-1262$. DOI: $10.1049 /$ ietcom. 2017.1436

[12] QIU, J., CHEN, L., LIU, S. A novel concatenated coding scheme: RS-SC-LDPC codes. IEEE Communication Letter, 2020, vol. 24 , no. 10, p. 2092-2095. DOI: 10.1109/LCOMM.2020.3004917

[13] AL-MOLIKI, Y. M., ALDHAEEBI, M. A., ALMWALD, G. A., e al. The performance of RS and RSCC coded cooperation systems using higher order modulation schemes. In Proceedings of 6 th International Conference on Intelligent Systems, Modelling and Simulation. Kuala Lumpur (Malaysia), 2015, p. 211-214. DOI: 10.1109/ISMS.2015.11

[14] ALMAWGANI, A. H. M., SALLEH, M. F. M. RS coded cooperation with adaptive cooperation level scheme over multipath Rayleigh fading channel. In Proceedings of IEEE 9th Malaysia International Conference on Communications (MICC). Kuala
Lumpur (Malaysia), 2009, p. 480-484. DOI: 10.1109/MICC.2009.5431555

[15] ALMAWGANI, A. H. M., SALLEH, M. F. M. Coded cooperation using Reed Solomon codes in slow fading channel. IEICE Electronics Express, 2010, vol. 7, no. 1, p. 27-32. DOI: 10.1587/elex.7.27

[16] MUGHAL, S., YANG, F., XU, H., et al. Coded cooperative spatial modulation based on multi-level construction of polar code. Telecommunication Systems, 2019, vol. 70, p. 435-446. DOI: $10.1007 / \mathrm{s} 11235-018-0485-6$

[17] BARRY, J. R., LEE, E. A., MESSERSCHMITT, D. G. Digital Communication. 3rd ed. Springer US, 2004. ISBN: 978-1-46150227-2

[18] BASAR, E., AYgOlU, U., PANAYIRCI, E., et al. New trellis code design for spatial modulation. IEEE Transactions on Wireless Communications, 2011, vol. 10, no. 8, p. 2670-2680. DOI: 10.1109/TWC.2011.061511.101745

\section{About the Authors ...}

Chunli ZHAO (corresponding author) received her B.S. degree in Electronic and Information Engineering from Henan Normal University Xinlian College, China, in 2014. She obtained her M.Sc. degree in Circuits and Systems at Henan Normal University, China, in 2017. She is currently doing Ph.D. from the College of Electronic and Information Engineering, Nanjing University of Aeronautics and Astronautics, China. Her research interests are circuits and systems, signal processing and channel coding.

Fengfan YANG received the M.Sc. and Ph.D. degrees from the Northwestern Polytechnical University and South-east University, China in 1993, and 1997, respectively, all in Electronic Engineering. He has been with the College of Information Science and Technology, Nanjing University of Aeronautics and Astronautics since May 1997. From October 1999 to May 2003, he was a research associate at the Centre for Communication Systems Research, University of Surrey, UK, and Dept. of Electrical and Computer Engineering, McGill University, Canada. His major research interests are information theory, channel coding and their applications for mobile and satellite communications.

Daniel Kariuki WAWERU received his B.Sc. degree in Mathematics and M.Sc. degree in Pure Mathematics from the University of Nairobi, Kenya, in 2015 and 2017. He is currently doing Ph.D. from the College of Electronic and Information Engineering, Nanjing University of Aeronautics and Astronautics, China. His research interests are coding theory, information theory, channel coding for wireless communications. 